\title{
Quaternaire
}

vol. 20/3 (2009)

Quaternaire et préhistoire ancienne de Normandie

Dominique Cliquet, Norbert Mercier, Jean-Pierre Lautridou, Philippe Alix, Valerie Beugnier, Romain Bianchini, Jean-Paul Caspar, Sylvie Coutard, Eric Lasseur, Pierre Lorren, Renaud Gosselin, Jean-Jacques Rivard et Helene Valladas

\section{Un atelier de production et de consommation d'outils bifaciaux de la fin du Paléolithique moyen à Saint- Brice-sous-Rânes (Orne, France) dans son contexte environnemental}

\begin{abstract}
Avertissement
Le contenu de ce site relève de la législation française sur la propriété intellectuelle et est la propriété exclusive de l'éditeur.

Les œuvres figurant sur ce site peuvent être consultées et reproduites sur un support papier ou numérique sous réserve qu'elles soient strictement réservées à un usage soit personnel, soit scientifique ou pédagogique excluant toute exploitation commerciale. La reproduction devra obligatoirement mentionner l'éditeur, le nom de la revue, l'auteur et la référence du document.

Toute autre reproduction est interdite sauf accord préalable de l'éditeur, en dehors des cas prévus par la législation en vigueur en France.
\end{abstract}

revues.org

Revues.org est un portail de revues en sciences humaines et sociales développé par le Cléo, Centre pour l'édition électronique ouverte (CNRS, EHESS, UP, UAPV).

Référence électronique

Dominique Cliquet, Norbert Mercier, Jean-Pierre Lautridou, Philippe Alix, Valerie Beugnier, Romain Bianchini, JeanPaul Caspar, Sylvie Coutard, Eric Lasseur, Pierre Lorren, Renaud Gosselin, Jean-Jacques Rivard et Helene Valladas, « Un atelier de production et de consommation d'outils bifaciaux de la fin du Paléolithique moyen à Saint-Bricesous-Rânes (Orne, France) dans son contexte environnemental », Quaternaire [En ligne], vol. 20/3 | 2009, mis en ligne le 01 septembre 2012, consulté le 06 juin 2014. URL : http://quaternaire.revues.org/5256 ; DOI : 10.4000/ quaternaire. 5256

Éditeur : Association Française pour l'Étude du Quaternaire

http://quaternaire.revues.org

http://www.revues.org

Document accessible en ligne sur : http://quaternaire.revues.org/5256

Ce document est le fac-similé de l'édition papier.

(c) Tous droits réservés 


\title{
UN ATELIER DE PRODUCTION ET DE CONSOMMATION D'OUTILS BIFACIAUX DE LA FIN DU PALÉOLITHIQUE MOYEN À SAINT-BRICE-SOUS-RÂNES (ORNE - FRANCE) DANS SON CONTEXTE ENVIRONNEMENTAL
}

\author{
Dominique CLIQUET ${ }^{1}$, Norbert MERCIER ${ }^{2 *}$, Jean-Pierre LAUTRIDOU ${ }^{3}$, \\ Philippe ALIX ${ }^{4}$, Valérie BEUGNIER ${ }^{5}$, Romain BIANCHINI ${ }^{6}$, Jean-Paul CASPAR $(\dagger)^{5}$, \\ Sylvie COUTARD ${ }^{6}$, Eric LASSEUR ${ }^{7}$, Pierre LORREN ${ }^{7}$, Renaud GOSSELIN ${ }^{8}$, \\ Jean-Jacques RIVARD ${ }^{7}$ \& Hélène VALLADAS ${ }^{2}$
}

\begin{abstract}
RÉSUMÉ
La mise en évidence, dans les années 1970, des stations de Saint-Brice-sous-Rânes, à l'occasion de ramassages de surface, a motivé une série de sondages visant à tenter de définir le potentiel subsistant de ces sites, puis la conduite d'une fouille d'une des nappes de vestiges. Le matériel collecté, presqu'exclusivement en silex, comporte principalement de nombreuses pièces bifaciales associées à une multitude d'éclats de façonnage. Une seconde série lithique uniquement constituée de produits de débitage a été reconnue. La méthode Levallois y est bien exprimée, à la différence de ce qui peut être observé dans les assemblages à outils bifaciaux. Le faible bilan sédimentaire qui caractérise ces sites du Grand Ouest reconnus en Bretagne, en Pays-dela-Loire et dans les deux Normandie n'autorise que rarement une interprétation chronostratigraphique de ces occupations. Cependant, la mise au jour d'artefacts chauffés sur le site de Saint-Brice a permis, pour la première fois dans le Grand-Ouest, de dater par les méthodes radiométriques un site d'atelier d'outils bifaciaux. Les dates obtenues (stades 6 et 3 de la chronologie isotopique) nous renvoient, pour l'occupation la plus récente, vers une problématique inhérente aux derniers peuplements néandertaliens d'Europe septentrionale et vers l'émergence des cultures à pièces bifaciales souvent foliacées de la fin du Paléolithique moyen. En effet, les ateliers de production d'outils bifaciaux du Grand-Ouest présentent quelques affinités avec les sites d'Europe moyenne et centrale.
\end{abstract}

Mots-clés : Paléolithique moyen, outils bifaciaux, stades isotopiques 6 et 3.

\section{ABSTRACT}

A BIFACIAL TOOL WORKSHOP AT THE END OF THE MIDDLE PALAEOLITHIC AT SAINT-BRICE-SOUS-RANES (ORNE - FRANCE) IN ITS ENVIRONMENTAL CONTEXT

The discovery, through fieldwalking during the seventies, of the Saint-Brice-sous-Rânes sites led to a series of test pits in order to define the site potential. This was followed by the partial excavation of an artifact layer. The assemblage, composed almost exclusively of flint artifacts, consists mainly of numerous bifaces and bifacial tools. A second lithic series constituted solely of knapping flakes, has been identified. The Levallois method is there well represented, contrary to the other series. The weak sedimentary record which characterizes sites of the western regions: Britanny, Loire region, Upper and Lower Normandy, rarely allow a chronostratigraphic interpretation of these sites. However, several heated flints at Saint-Brice allow, for the first time in the western regions, the dating through radiometric methods of a biface workshop. The ages obtained (MIS 6 and 3 ) show that this is a site of the last Neanderthalians of northern Europe and confirm the emergence of bifacial tool cultures in western France at the end of the Middle Palaeolithic. In fact the bifaces workshops in the western France have some similarities with the central and middle Europe sites.

Keys-words: Middle Palaeolithic, bifacial tools, MIS 6 and 3

\footnotetext{
${ }^{1}$ Service régional de l'Archéologie, Direction régionale des Affaires culturelles de Basse-Normandie et PCR «Les Premiers Hommes en Normandie», 13 bis, rue Saint-Ouen, F-14052 Caen cedex 04 / UMR 6566 CNRS, Université de Rennes 1. Courriel: dominique.cliquet@culture.gouv.fr ${ }^{2}$ Laboratoire des Sciences du Climat et de l'Environnement, Unité mixte CNRS-CEA, Avenue de la Terrasse, F-91198 Gif-sur-Yvette Cedex. ${ }^{3}$ M2C, C.N.R.S. - U.M.R. 6143 - Morphodynamique continentale et côtière, Centre de géomorphologie, rue des Tilleuls, F-14 000 Caen et PCR «Les Premiers Hommes en Normandie», Direction régionale des Affaires culturelles de Basse-Normandie, 13 bis, rue Saint-Ouen, F-14052 Caen cedex 04 ${ }^{4}$ Institut national de Recherches archéologiques préventives, Rhône-Alpes, 12, rue Maggiorini, F-69 500 Bron et PCR «Les Premiers Hommes en Normandie », Direction régionale des Affaires culturelles de Basse-Normandie, 13 bis, rue Saint-Ouen, F-14052 Caen cedex 04

${ }_{5}^{5}$ Prehistoric Archaeology Unit, Department of Geography and Geology, Katholieke Universiteit Leuven, Celestijnenlann 200, B-3001 Heverlee

${ }^{6}$ Institut national de Recherches archéologiques préventives, Nord-Picardie, 518, rue Saint-Fuscien, F-80 000 Amiens et PCR «Les Premiers Hommes en Normandie», Direction régionale des Affaires culturelles de Basse-Normandie, 13 bis, rue Saint-Ouen, F-14052 Caen cedex 04

${ }^{7}$ PCR «Les Premiers Hommes en Normandie», Direction régionale des Affaires culturelles de Basse-Normandie, 13 bis, rue Saint-Ouen, F-14052 Caen cedex 04

812 rue Morère, F-75 014 Paris

* Nouvelle adresse: Institut de recherche sur les Archéomatériaux, UMR 5060 CNRS, Université Bx3, Centre de Recherche en Physique Appliquée à

l'Archéologie (CRP2A), Maison de l'archéologie, F-33607 Pessac cedex. Courriel : Norbert.Mercier@u-bordeaux3.fr
} 


\section{1 - LOCALISATION DU SITE ET HISTORIQUE DES OPÉRATIONS}

La commune de Saint-Brice-sous-Rânes se situe, dans le département de l'Orne au sud-ouest d'Argentan vallée de l'Orne. Ce site se caractérise par un abondant matériel réparti sur d'importantes surfaces (plusieurs hectares) et correspond notamment à des ateliers de production (construction du volume bifacial, confection) et de consommation (utilisation et entretien) d'outils bifaciaux. L'industrie se localise sur un plateau étroit, relativement plan, non disséqué dans la zone concernée, à 235 - $249 \mathrm{~m}$ d'altitude (fig. 1).

Les investigations menées et programmées, depuis 1998, permettent d'appréhender l'étendue du complexe d'ateliers, d'une part, et d'analyser une aire d'occupation paléolithique encore en place, d'autre part. Rappelons que deux concentrations denses de vestiges ont été mises en évidence, dont l'une a fait l'objet d'une fouille partielle.

Cette nappe de vestiges, grossièrement elliptique, couvre une superficie d'environ $500 \mathrm{~m}^{2}$, dont «l'épicentre» a été fouillé. En effet, les sondages en tranchées réalisés ensuite ont révélé l'existence de gradients de vestiges (fig. 2).

\section{2 - CONTEXTE GÉOLOGIQUE ET GÉOMORPHOLOGIQUE DU SITE}

Sous une couche d'épaisseur irrégulière de lœss $(0,5$ à $2 \mathrm{~m}$ ) altéré sur $1 \mathrm{~m}$ par le sol de surface (sol brun lessivé avec un $\mathrm{Bt}$ à tendance hydromorphe: marbrures et taches grises, concrétions ferro-manganiques) se trouve une couche à cailloux de silex et de calcaire silicifié et à pisolithes ferrugineux.

Ces deux formations reposent sur l'argile silteuse grise jurassique (Toarcien) de 3 à $4 \mathrm{~m}$ d'épaisseur, rubéfiée sur $60 \mathrm{~cm}$ ainsi que la couche à cailloux (fig. 3). Cette argile recouvre des sables grésifiés du Jurassique inférieur (Pliensbachien; Kuntz et al., 1989), d'épaisseur variable (quelques mètres), et au contact avec le granite, un cailloutis de base du Jurassique à sables et galets qui affleurent, remaniés sur le versant à l'ouest du Bois de Rânes.

L'argile silteuse toarcienne (Lias) est recouverte plus au nord (vallée de l'Orne) par des calcaires jurassiques comportant de bas en haut un calcaire bioclastique peu épais (Bajocien), le Calcaire à silex d'Ecouché et le Calcaire oolithique de Sarceaux du Bathonien moyen et le Calcaire fin d'Argentan du Bathonien supérieur. Ces calcaires ont recouvert le plateau de Rânes et plus au sud, partiellement, le massif granitique de la Ferté Macé, puis ont fondu par altération.

\section{1 - OBSERVATIONS GÉNÉRALES ET ÉTUDE DE LA «COUCHE Á CAILLOUX»}

Les observations effectuées durant les campagnes de fouilles et de sondages ont porté sur le même plateau.

Plusieurs sondages et excavations encore ouvertes dans le Bois de Rânes témoignent de remaniements anthropiques sur plus d'un mètre de profondeur (mélange

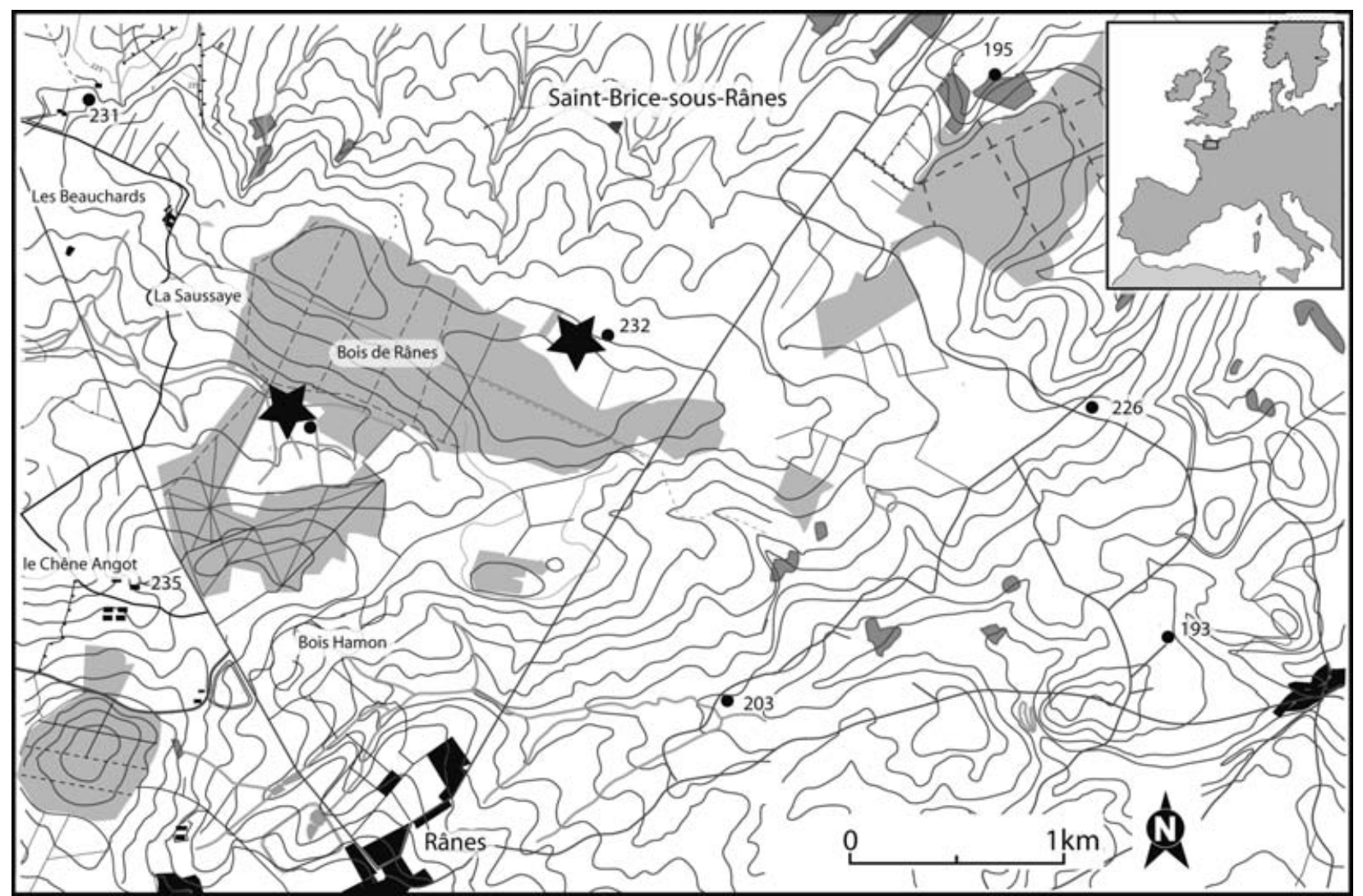

Fig. 1 : Localisation du site.

Fig. 1: Location map. 


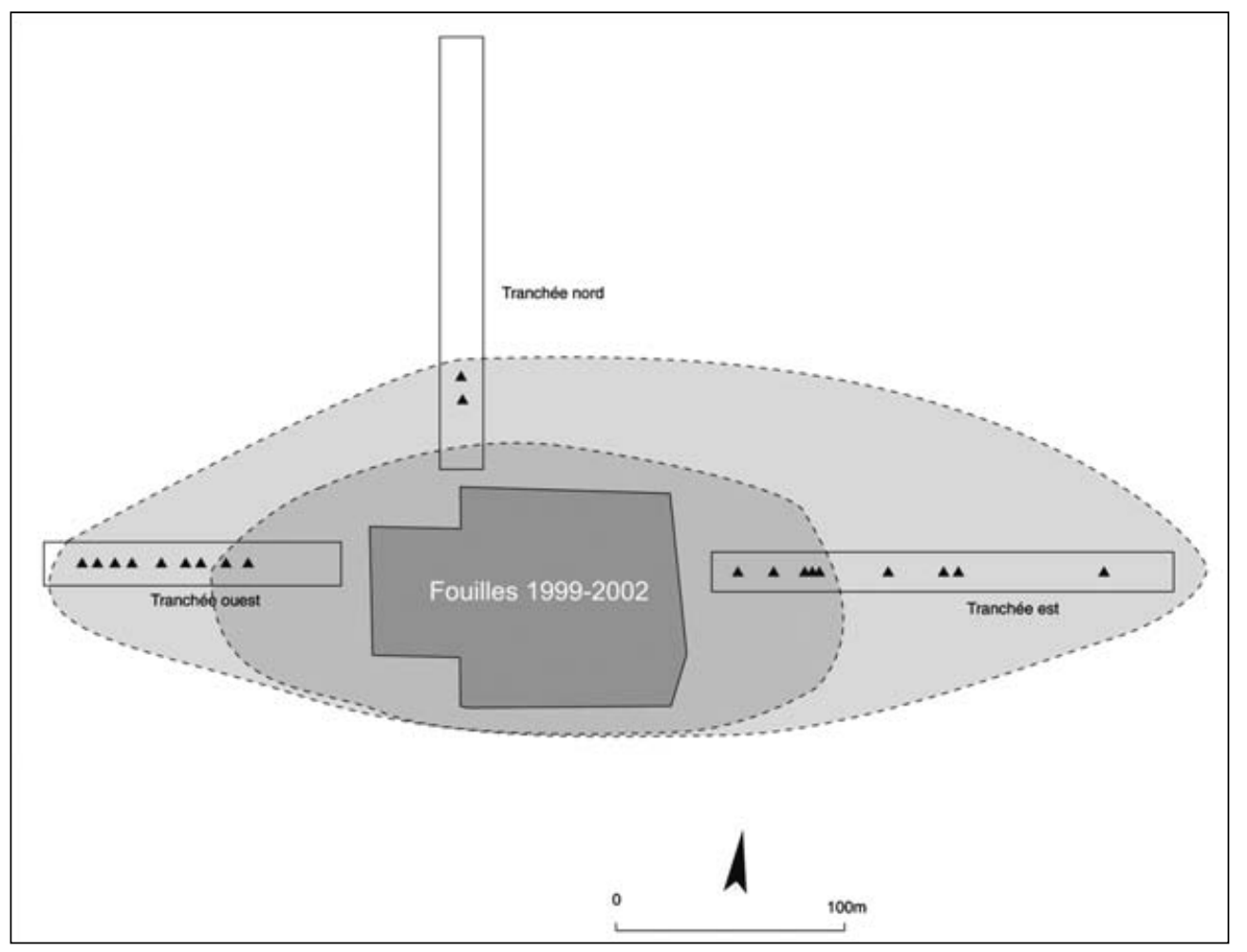

Fig. 2 : Morphologie de la nappe de vestiges du site P6 de Saint-Brice-sous-Rânes. Fig. 2: Morphology of the P6 Saint-Brice-sous-Rânes site artifact layer.

de limon jaune, de limon humifère, charbon de bois, scories), correspondant à la recherche de minerai de fer.

En bordure de ce bois, une fosse, profonde de quatre mètres a été creusée puis remblayée par l'homme aux
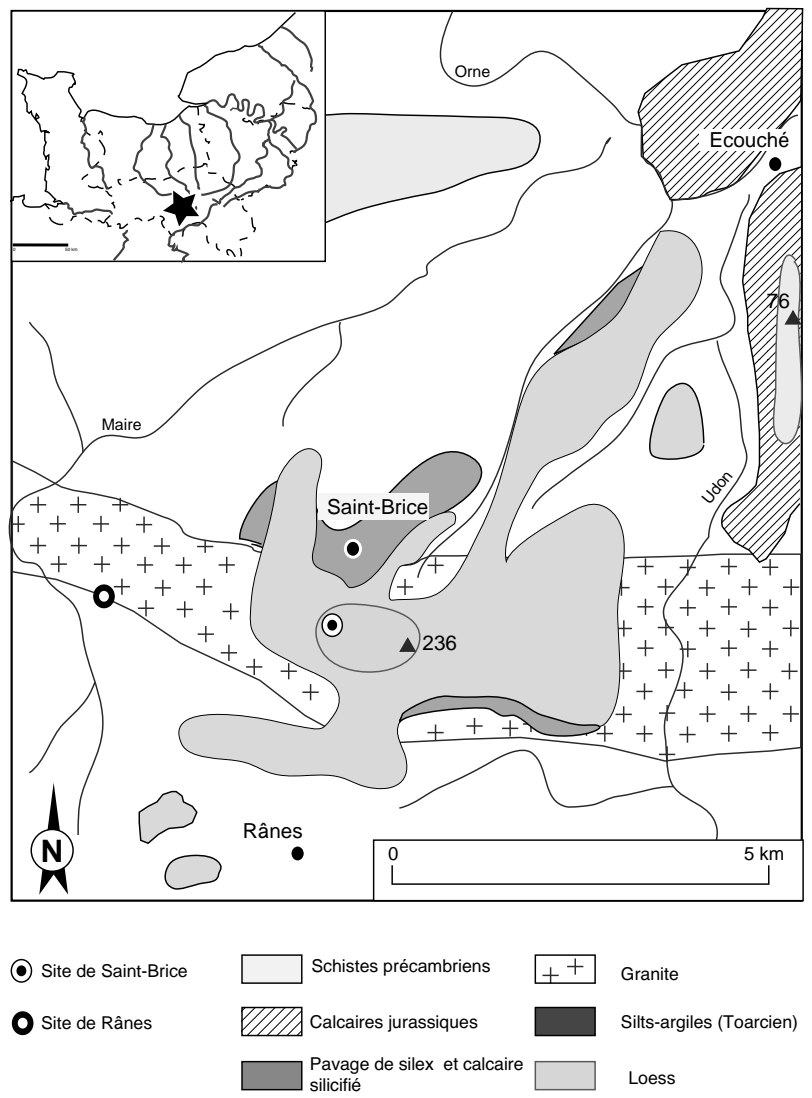

Fig. 3 : Croquis morphologique du site.

Fig. 3: Morphological diagram. périodes moderne ou contemporaine. Outre le remplissage anthropique précédemment signalé on a trouvé plusieurs dalles $(50 \mathrm{~cm})$ de silex d'Ecouché et un gros bloc $(40 \mathrm{~cm})$ de cuirasse ferrugineuse. Vraisemblablement ce reste de cuirasse ferrugineuse latéritique devait être abondant dans le Bois de Rânes et exploité en tant que minières. La cuirasse a aggloméré des fragments de calcaire oolithique silicifié de Sarceaux. On la retrouve démantelée dans la couche à cailloux sous forme de granules et pisolithes ferrugineux.

Cette couche résulte d'une longue histoire depuis le début du Crétacé jusqu'au Tertiaire moyen, avec plusieurs étapes: silicification du calcaire jurassique (calcaire silicifié), fonte du calcaire (Argentan, Sarceaux, Ecouché) laissant un peu d'argile à silex et surtout un pavage de silex (d'Ecouché) et de calcaire silicifié, formation d'une cuirasse ferrugineuse et silicification de sables venus du massif ancien (grès) sans doute à l'Eocène dont on ne trouve que peu de restes. Ce sont: un bloc au nord-ouest de la feuille Argentan, quelques blocs au sud-est sur les grès primaires à Bois-1'Evêque qui constitue l'avancée nord de la Forêt d'Ecouves qui domine toute la région avec des altitudes supérieures à $300 \mathrm{~m}$.

Un grès a été taillé par les Paléolithiques. On ne peut pas différencier dans le temps l'étape «cuirasse» et l'étape «grès». Cependant, dans les deux cas, les calcaires silicifiés ont été inclus dans les deux processus de formation d'une cuirasse et de silicification.

Des restes de grès et de cuirasses sont signalés au nord (Forêt de Gouffern) et au Sud (Ferté-Macé). Les calcaires silicifiés sont présents, toujours associés au pavage de silex (couche à cailloux) autour de Rânes. On 
les signale à l'ouest (Carte géologique de Flers) et au nord-ouest (Campagne de Caen).

L'ouverture principale a montré que le pavage grossier était constitué pour moitié de ces fragments de calcaire silicifié, l'autre moitié provenant des silex du Calcaire d'Ecouché.

Pendant le reste du Tertiaire et au Quaternaire le pavage (couche à cailloux) a été démantelé, affecté par le gel. Toutefois on n'observe pas de structure périglaciaire alors qu'en raison de «l'altitude» il est très probable que pendant le maximum de chaque période froide un pergélisol affectait les zones élevées.

Cela s'explique par le fait que le substrat d'argile silteuse est très défavorable à l'expression de structures périglaciaires: ainsi en Arctique il n'y a pas sur l'argile de grandes fentes de gel (ice wedges) qui sont des marqueurs de pergélisol; les structures de cryoturbation nécessitent un matériel moins fin et surtout plus hétérogène. L'ouverture principale a permis d'observer des ondulations de la couche à cailloux et pisolithes sous forme de bosses typiques: des «thufurs», étudiés notamment en Islande et en Arctique, qui témoignent d'une activité périglaciaire; mais cette figure existe en région froide avec ou sans pergélisol.

\section{2 - LE MICRO-MODÈLE EN BOSSES DE LA COUCHE À CAILlOUX DE SILEX, CALCAIRE SILICIFIÉ, PISOLITHES FERRUGINEUX (PAVAGE)}

Le modelé des bosses a donc été mis en évidence sur le site fouillé (fig. 4). Le sommet de chaque bosse est $30 \mathrm{~cm}$ plus haut que le creux; les bosses constituent un champ de petits monticules répartis irrégulièrement. Parfois, deux bosses sont reliées entre elles. La distance entre le sommet de deux bosses est de 1 à $1,2 \mathrm{~m}$.

Ces caractéristiques sont celles des «thufurs》 des régions froides. Les bosses sont enrichies en glace avec parfois un noyau de glace. Lors de l'engel saisonnier la glace se répartit irrégulièrement dans la couche à cailloux en fonction de différences parfois minimes de la granulométrie (donc de la porosité). Il se constitue ainsi des champs de thufurs.

Lors de la mort de ces structures (avec fonte de la glace) pour une raison quelconque (par exemple succession de plusieurs étés plus chauds) on ne retrouve pas la topographie initiale plate. La bosse s'affaisse partiellement mais pas totalement (circulation de matériel fin remplaçant en partie les lentilles de glace). À la différence des figures de cryoturbation il n'y a pas de structures de forte pression, mais un simple soulèvement du matériel par des lentilles de glace.

Au début de l'engel automnal les cailloux de surface gèlent plus vite que la matrice. Une lentille de glace qui grossit régulièrement par cryosuccion de l'eau de la matrice non encore gelée, soulève les cailloux qui tendent à se redresser verticalement pour obtenir une expulsion optimum.

Ce phénomène qui existe encore actuellement en Normandie lors d'hivers très froids explique la concentration, bien connue des agriculteurs, des cailloux en

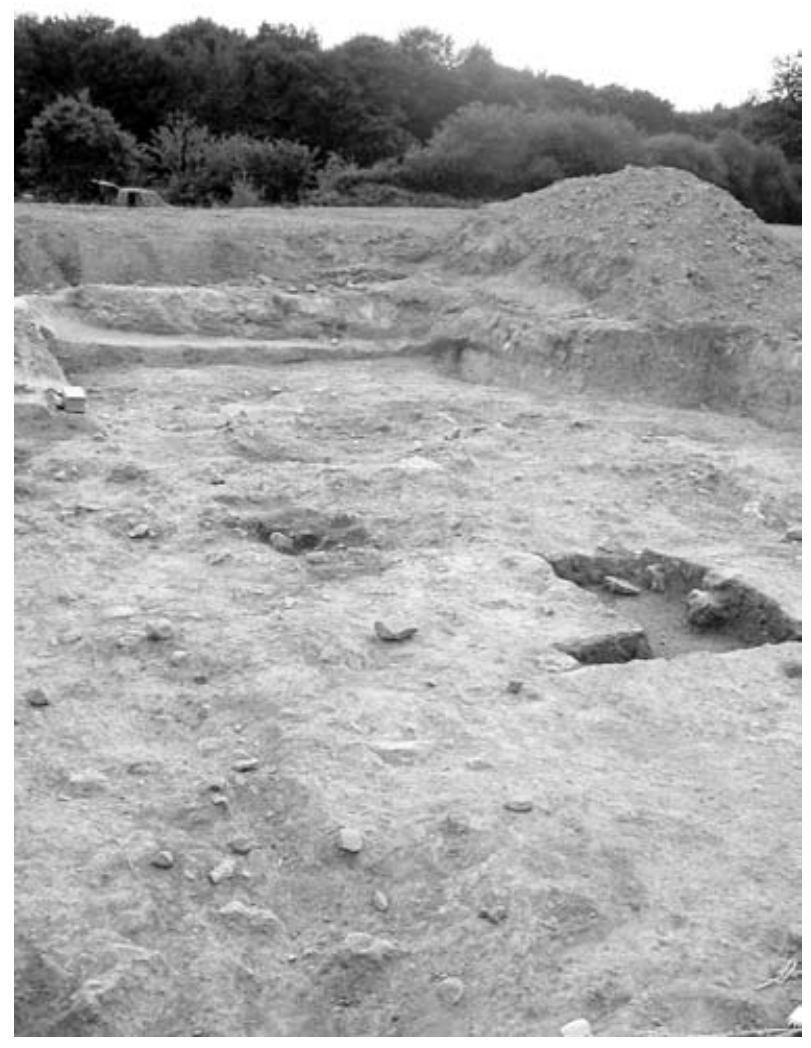

Fig. 4 : Micro-modelé en bosses de la couche à cailloux (Cliché D. Cliquet) - (cf. PI. V, fig. 5b, p. 386).

Fig. 4: Bump micro-model of the pebble layer (Photo D. Cliquet) (cf. Pl. V, fig. 5b, p. 386).

surface (les cailloux «poussent»). Ce processus de "frost heave» permet de comprendre la présence de cailloux et de silex taillés en position oblique à sub-verticale sur certaines bosses.

\section{3 - UN FAIBLE BILAN SÉDIMENTAIRE}

Les observations effectuées sur le site fouillé et dans les sondages ont permis de distinguer cinq formations, comportant de haut en bas (fig. 5):

1) loess avec le sol de surface $(0-140 \mathrm{~cm}): 0-40 \mathrm{~cm}$ horizon humifère Ap., 40-100 cm B textural limono-argileux marron à marbrures grises. De 100 à $140 \mathrm{~cm}$ : lœss brun jaune qui peut atteindre un mètre ou plus d'épaisseur dans les autres sondages.

2) limon argileux brun légèrement rougeâtre remplissant les creux entre les bosses (140-170 cm). Argileux à la base il devient progressivement limoneux (lœssique). L'argile provient d'apports latéraux (argile des bosses) et d'illuviations au sein du sédiment, le lœss devient prédominant vers le haut: c'est la base de la séquence loessique comprimée et lacunaire de Saint-Brice avec, au début, un mélange de loss et de matériel local (couche 2) comme dans les limons bruns feuilletés des plateaux de Normandie (Lautridou, 1985), puis, un lœss weichselien (couche 1).

$3)$ couche à cailloux $(170-185 \mathrm{~cm})$. Matrice argileuse rougeâtre (5YR) avec des cailloux de toutes tailles $(0,5$ à parfois $30 \mathrm{~cm}$ ): silex gris gélifractés d'Ecouché, cailloux très abondants, de forme légèrement arrondie, de calcaire silicifié à cortex rougeâtre ferrugineux (de 1 à $10 \mathrm{~cm}$ en 

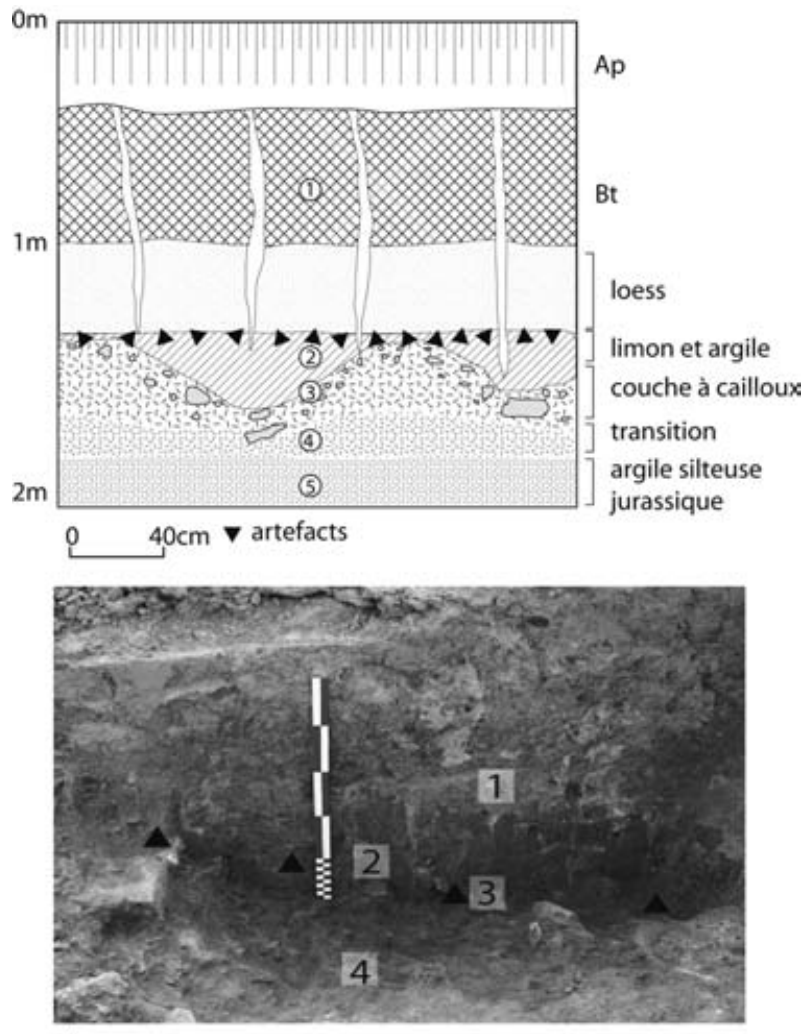

A industrie lithique

Fig. 5: Bilan sédimentaire (cf. Pl. V, fig. 5a, p. 386).

Fig. 5: Sedimentary record (cf. Pl. V, fig. 5a, p. 386).

général), granules et pisolithes ferrugineux $(0,5$ à $3 \mathrm{~cm})$ abondants. Les sept premiers centimètres supérieurs sont riches en éclats (gel) de silex (de 1 à $2 \mathrm{~cm}$ ). Une lame mince réalisée dans la partie supérieure de ce cailloutis a montré des traits cryogéniques bien marqués, à savoir d'épaisses coiffes silteuses sur les graviers et une ségrégation des particules limoneuses dans la masse basale.
4) argile rougeâtre $(185-200 \mathrm{~cm})$ à nombreux granules ferrugineux.

5) argile gris-jaune encore légèrement rougeâtre au sommet $(200 \mathrm{~cm})$. On ne distingue pas de véritable coupure ni entre 1 et 2 , ni entre 3 et 4 , pas plus qu'entre 4 et 5 .

L'argile de la couche à cailloux (3) et de l'horizon (4) est l'argile toarcienne (5) qui a subi de nombreux brassages au Tertiaire (après l'Eocène) et au Quaternaire avec altération chimique (Tertiaire, interglaciaires du Quaternaire), rubéfaction, démantèlement de la cuirasse ferrugineuse, du pavage de silex, localement des silifications éocènes. Dans cette zone plate il n'y a pas eu de véritables déplacements latéraux (ruissellement, gélifluxion...).

Les sédiments de la couche (2) observés en lame mince portent la marque de plusieurs pédogenèses (tab. 1). Trois phases d'illuviation sont nettement marquées. La première n'existe plus qu'à l'état de papules intégrées à la masse, elle témoigne de l'érosion d'un sol brun lessivé. La deuxième, sombre et microlitée, souvent argilo-humique pourrait correspondre à l'horizon d'accumulation d'un sol de type forestier. Enfin, la troisième, jaune orangée, correspond probablement à la pédogenèse holocène de surface.

Par corrélation avec les séquences décrites dans les régions voisines (Antoine in Locht, 2002; Locht et al., 2003 ; Antoine et al., 1999), ces traits pédo-sédimentaires pourraient donc témoigner de l'érosion du sol brun lessivé eemien, suivie d'une pédogenèse humifère de type sol forestier attribuable au Weichselien ancien .

Lors de la dernière période froide, des structures périglaciaires se sont développées avec formation de bosses (thufur), permettant la préservation (limitée) de limons argileux contenant des reliques de pédogenèses eemiennes et weichseliennes, avant le dépôt de lœss allochtones.

\begin{tabular}{|c|c|c|}
\hline Echantillon & Couche & Traits pédosédimentaires \\
\hline Saint-Brice 1 & poche de limon (2) & $\begin{array}{l}\text { fond silto-sableux quartzeux } \\
\text { illuviation très abondante: } \\
\text { 1. nombreuses petites papules argileuses roulées intégrées dans la } \\
\text { masse basale. } \\
\text { 2. nombreux revêtements brun foncé à acajou microlités épais } \\
\text { déconnectés de la porosité active, souvent argilo-humiques. } \\
\text { 3. revêtements jaune orangé peu fréquents dans la porosité }\end{array}$ \\
\hline Saint-Brice 3 & poche de limon (2) & $\begin{array}{l}\text { silt quartzeux et forte fraction sableuse (grains de quartz souvent } \\
\text { émoussés) } \\
\text { illuviation : } \\
\text { 1. agrégats arrondis à matrice plus foncée, plus poussiéreuse, entourés et } \\
\text { parfois recoupés par l'illuviation, contenant de nombreuses papules } \\
\text { rougeâtres (= pédoreliques) } \\
\text { 2. illuviation brune poussiéreuses éparse. } \\
\text { 3. argileuse massive orangée, claire, limpide à légèrement poussiéreuse, } \\
\text { occupant des chenaux. } \\
\text { remplissage de cavités biologiques silto-argileux stratifié avec passées } \\
\text { silto-humiques assez foncées microlitées } \\
\text { structure lamellaire discontinue plus ou moins marquée } \\
\text { localement, hyporevêtements ferro-manganiques } \\
\text { plages lavées (hydromorphie) }\end{array}$ \\
\hline Saint-Brice 2 & cailloutis (3) & $\begin{array}{l}\text { illuviation argileuse fine orangée foncé dans les chenaux et les pores } \\
\text { inter-granulaires, parfois microlitée dans les plus gros pores où l'épaisseur } \\
\text { des revêtements est plus grande. Cette illuviation n'est pas présente } \\
\text { partout. } \\
\text { coiffes silteuses sur les graviers, bien développées (trait cryogénique) }\end{array}$ \\
\hline
\end{tabular}

Tab. 1 : Traits pédosédimentaires présents dans les trois échantillons étudiés.

Tab. 1: Pedosedimentary characteristics present in the three sediment samples tested. 
La nappe de vestiges anthropiques se situe au sommet de la couche 2 jusqu'au toit des bosses avant le dépôt du lœss 1 allochtone (fig. 6).

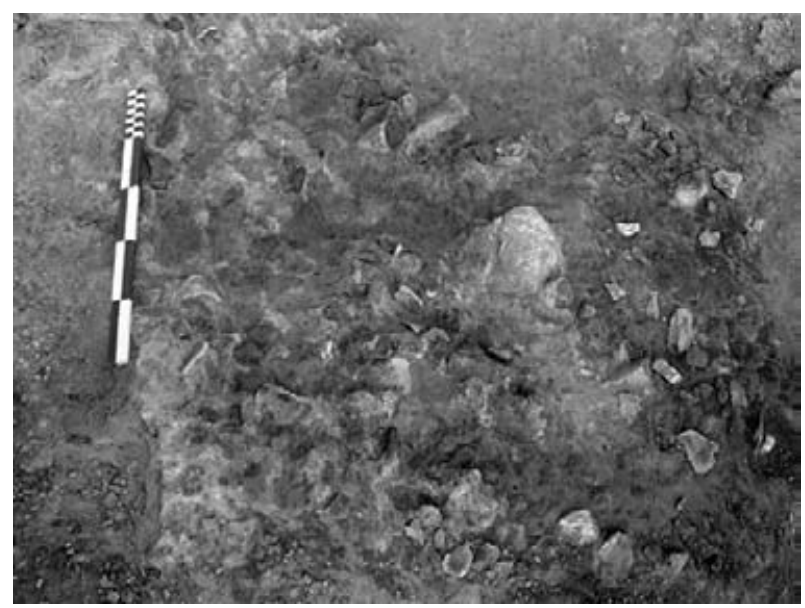

Fig. 6: Nappe de vestiges (Cliché D. Cliquet) - (cf. PI. VI, fig. 6a, p. 387). Fig. 6: Artifact layer (Photo D. Cliquet) - (cf. Pl. VI, fig. 6a, p. 387).

Une autre coupe, proche du site et moins profonde, donne une image plus irrégulière de la couche à cailloux. Comme dans la coupe précédente et comme dans le site fouillé, certains gros blocs pénètrent dans la couche 3 . Sur la fouille, plusieurs cas de blocs situés au sommet de bosses ont été observés. Certains blocs de silex de la couche à cailloux sont gélifractés sur place, sans déplacement ultérieur.

Ces éléments plaident en faveur d'un niveau d'occupation rapportable à la phase récente du Paléolithique moyen et plus vraisemblablement au Pléniglaciaire inférieur ou moyen.

\section{3 - LES OCCUPATIONS HUMAINES DU SITE DE SAINT-BRICE-SOUS-RÂNES}

Le matériel lithique collecté en surface à Saint-Bricesous-Rânes comporte un ensemble d'éléments qui pourraient attester, dans l'état actuel de la recherche, de la présence d'au moins deux occupations diachroniques:

- une série à patine blanche, caractérisée par un débitage Levallois de grande dimension, sans biface. Cet ensemble a fait l'objet de sondages dans le but de préciser la situation topographique de l'occupation, la chronostratigraphie de l'ensemble et, si possible, de préciser une chronologie relative par rapport à l'assemblage caractérisé par l'abondant outillage bifacial, énoncé ci-dessus. Les investigations n'ont pas permis de reconnaître de niveaux archéologiques en place. L'aire de distribution de ce matériel en surface a été entièrement déstructurée aux périodes moderne et contemporaine par le creusement de minières destinées à alimenter les nombreuses forges, dans l'espace évalué.

Cependant, la présence de quelques éléments très fragmentés par le gel associés au «cailloutis» qui constitue les thufur(s) témoignerait de l'antériorité de cette «série blanche» par rapport au second ensemble.
En effet, la présence d'éléments susceptibles d'appartenir à cet ensemble a été notée à plusieurs reprises sur l'espace de production d'outils bifaciaux. Ce mobilier, toujours très affecté par le gel, se retrouve systématiquement associé au cailloutis à la base. Ces quelques pièces témoignaient donc de l'existence d'une occupation du site antérieure à la mise en place du «pavage». Son âge reste cependant incertain (Saalien?).

- un assemblage constitué d'une majorité d'outils à retouches bifaciales couvrantes et / ou envahissantes bifaces et outils bifaciaux - qui n'est pas sans rappeler les faciès à outils bifaciaux de l'Europe du Nord-Ouest et d'Europe centrale. Ce mobilier apparaît associé au limon argileux brun légèrement rougeâtre remplissant les creux entre les thufur(s).

C'est cet ensemble qui a principalement été l'objet de nos investigations.

\section{1 - LA «SÉRIE BLANCHE» À DÉBITAGE LEVALLOIS}

Les prospections de surface ont livré quelques artefacts à patine blanche localisés dans deux secteurs bien déterminés. Cependant, les sondages pratiqués n'ont pas permis de trouver ce mobilier en contexte chronostratigraphique. Si l'industrie se différencie très nettement des assemblages à outils bifaciaux, par son état physique (patine blanc-jaune mate), d'autres critères l'individualisent. En premier lieu, les artefacts présentent fréquemment des altérations dues au gel, en second lieu, le petit assemblage ne semble comporter que de rares pièces bifaciales et se caractérise par une production Levallois d'excellente facture, bien exprimée (fig. 7).

La matière première mise en œuvre est le silex local. L'assemblage lithique apparaît constitué d'éléments issus de chaînes de débitage orientées vers la production d'éclats prédéterminés, Levallois notamment. Les modalités de mise en œuvre sont illustrées par un débitage récurrent uni ou bipolaire majoritaire.

La préparation des surfaces de débitage et de plan de frappe fait l'objet d'un soin tout particulier, contrairement à ce qui a pu être observé dans les assemblages à outils bifaciaux.

L'outillage n'est actuellement représenté que par quelques racloirs, pour certains très altérés par le gel. L'hypothétique appartenance à cet ensemble d'un biface et de deux éclats de façonnage d'aspect physique similaire nécessiterait confirmation.

Les caractéristiques physiques et techniques de cet assemblage l'individualisent très bien du second ensemble.

\section{2 - CARACTÉRISTIQUES DE LA SÉRIE PRINCIPALE RAPPORTÉE AU PALÉOLITHIQUE MOYEN À OUTILS BIFACIAUX}

Largement représenté en surface, le Paléolithique moyen à outils bifaciaux a fait l'objet de nombreux sondages visant à mettre en évidence des niveaux en place. Deux nappes de vestiges ont été reconnues; l'une 

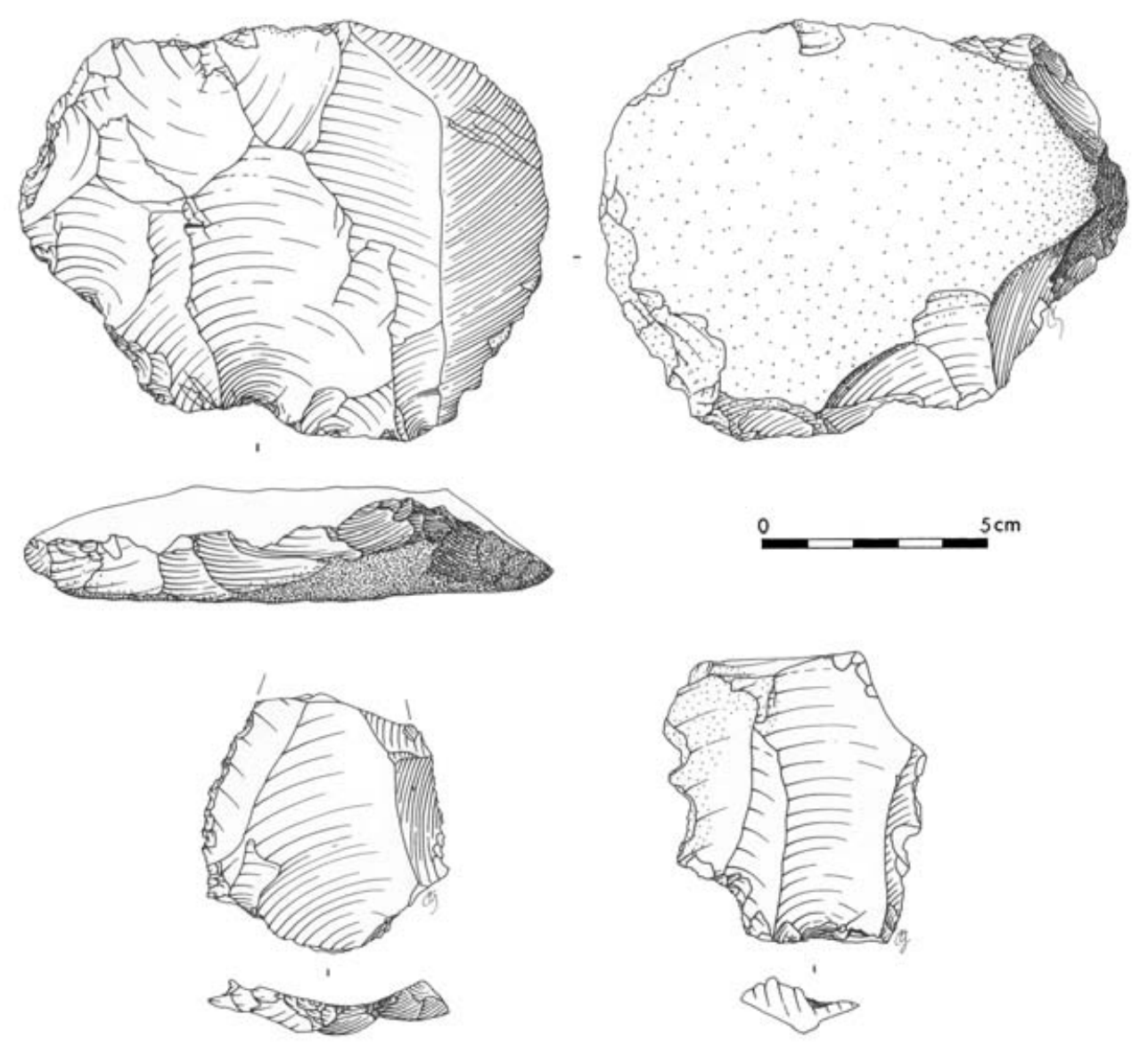

Fig. 7 : Industrie lithique de la «série blanche» (dessin P. Alix) Fig. 7: The "white series" lithic industry (drawing P. Alix).

d'elles a fait l'objet d'investigations durant cinq années consécutives (1998-2002). L'ouverture pratiquée, de petite dimension, environ $120 \mathrm{~m}^{2}$, a livré un très abondant matériel, soit plus de 100000 objets lithiques.

\subsection{1 - Les matières premières}

L'étude gîtologique des matières premières mises en œuvre sur le site de Saint-Brice est fondée sur une étude pétrologique des matériaux utilisés (microscopie optique et macroscopie), sur l'intégration à la réflexion des problèmes d'accessibilité de la matière d'œuvre pendant l'occupation, d'une part, de la morphologie, des modules et de l'aptitude à la taille des matériaux, d'autre part. La cartographie des données a permis une confrontation des matières premières, des matériaux utilisés par les Paléolithiques et des espaces investis durant l'occupation du gîte de matière première.

Il ressort en premier lieu, de cette analyse, que le silex est très nettement majoritaire (plus de $99 \%$ des artefacts collectés) et présente un aspect «frais». Il provient de l'argile à silex actuellement affleurante, issue de la décarbonatation des Calcaires d'Écouché, datés du Bathonien moyen (Kuntz et al., 1989; Coutard, 1998). La cartographie atteste d'une corrélation bien marquée entre les nappes de vestiges du Paléolithique moyen et les pavages à silex (Lasseur, 2001; Lasseur et al., 2005). Ainsi, les «gîtes potentiels» de matière d'œuvre sont situés sur des sommets de versant présentant une pente supérieure à $3^{\circ}$. Ces «zones» se caractérisent par une grande abondance de fragments de silex gélifractés de gros module présentant une bonne aptitude à la taille. Les plateaux quant à eux s'individualisent par une faible quantité d'éléments siliceux, constitués pour la plupart de nodules peu affectés par le gel.

L'étude technologique du mobilier témoigne de l'utilisation d'éclats de gel comme supports pour le façonnage (préformes) et de gros rognons parfois affectés par le gel. Le silex est donc prélevé à l'endroit même où il est mis en œuvre, comme sur de nombreux sites d'atelier.

Les ateliers se développent donc sur le versant nord du plateau, le moins accueillant pour une implantation des populations. D'ailleurs, les sondages n'ont jusqu'alors révélé aucune structuration susceptible d'évoquer un habitat. De rares pièces témoignent de l'utilisation de grès locaux et de galets de quartz.

\subsection{2 - Caractéristiques techniques et technolo- giques de l'industrie}

L'assemblage lithique issu de la fouille, fort de plus de 100000 artefacts, est essentiellement constitué «d'éclats de taille de biface», d'esquilles, d'éclats de régularisation ou de retouche, d'outils bifaciaux, où les pièces corticales apparaissent relativement peu nombreuses. Enfin, la série compte peu de nucléus (n: 128). 
La multitude des enlèvements, notamment de petites dimensions, atteste d'un débitage in situ, et illustre, les opérations de débitage (pièces d'aménagement de plans de frappe, abrasions de corniches...), les processus de façonnage (éclats de taille de biface, de retouche...), le réaménagement et l'entretien des outils (éclats de coup de tranchet, de retaille de biface, de retouche).

Le débitage Levallois est attesté, bien que peu représenté; les quelques enlèvements collectés sont issus d'un schéma opératoire récurrent centripète. Leur lecture n'est pas toujours aisée en contexte de façonnage de pièces bifaciales où les éclats de taille de biface apparaissent dominants. Ces derniers attestent de la production d'outils bifaciaux et de leur entretien (réaffûtage) sur le site même.

Au regard du nombre de pièces collectées, les artefacts retouchés s'avèrent peu nombreux. Les outils sont principalement aménagés sur des éclats, tant les outils «classiques» définis par Bordes (1961) que les pièces bifaciales. Aussi, la chaîne opératoire de façonnage semble souvent découler d'une chaîne opératoire de débitage, le plus souvent courte (production d'éclats corticaux, de supports kombewa...), ou «opportuniste», exploitant de grands éclats de gel présentant déjà les convexités naturelles («construction volumétrique de l'outil»). Cependant quelques racloirs ont été aménagés sur des éclats de façonnage d'outils bifaciaux.

L'observation des talons témoigne d'un certain soin apporté à la préparation des plans de frappe, particulièrement pour les éclats de taille de biface de fin de séquence (abrasion, doucissage, percussion tangentielle exercée au percuteur organique).

L'outillage «classique» sur éclat comporte donc des racloirs, des pièces affectées d'encoches, des grattoirs, un burin, auxquels s'ajoutent des pièces bifaciales (fig. 8), à savoir (Cliquet et al., 2001):

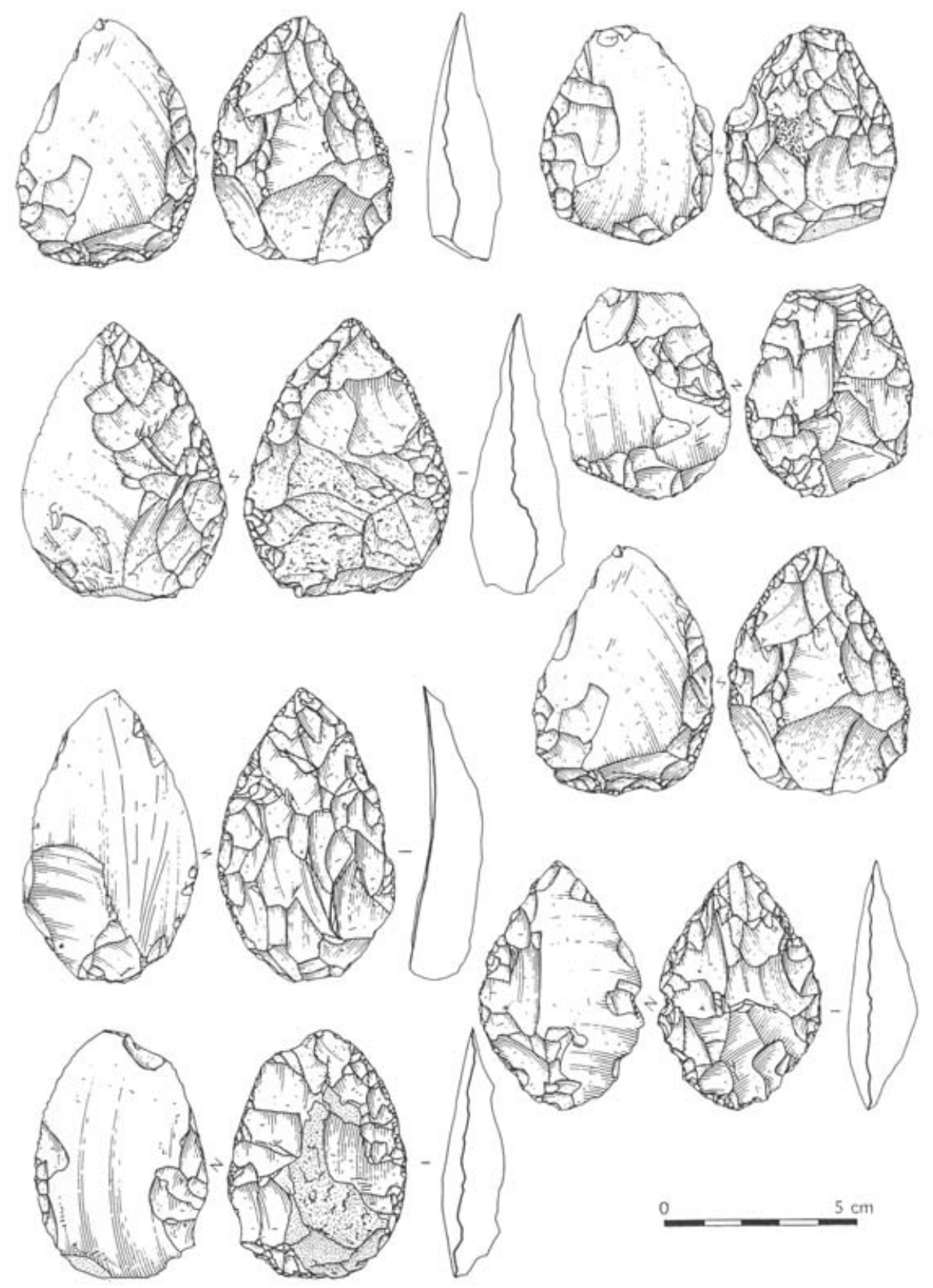

Fig. 8: Industrie lithique de la «série à outils bifaciaux» (dessin P. Alix).

Fig. 8: The "bifacial tool series" lithic industry (drawing P. Alix). 
- les outils bifaciaux;

- les «bifaces vrais» ou bifaces-outils qui conservent leur morphologie initiale malgré les réaffûtages successifs;

- les «bifaces supports d'outils» dont la forme évolue en fonction des aménagements dont ils sont affectés en cours d'utilisation. Ces derniers s'apparentent aux outils bifaciaux et présentent un état d'exhaustion plus avancé que les bifaces «vrais» au moment de l'abandon.

Ces outils sont de petites dimensions.

Malgré des résultats initialement prometteurs, l'analyse tracéologique ne pourra apporter sa contribution à l'analyse fonctionnelle du matériel. En effet, les observations effectuées, sur environ 120 pièces, par Jean-Paul Caspar $(\dagger)$, puis par Valérie Beugnier (Université de Louvain, Belgique) attestent d'une profonde altération des polis d'utilisation(s). Ce diagnostic va à l'encontre des observations effectuées courant 1999 par Renaud Gosselin (in Cliquet et al., 2001) sur une douzaine d'objets. Il est très regrettable que l'étude fonctionnelle ne puisse être tentée sur ce matériel. En effet, seul le recours à la tracéologie permettra de comprendre le système évolutif des pièces bifaciales; la technologie n'autorisant qu'un constat.

Par ailleurs, l'étude du mobilier atteste de la présence d'au moins trois types de pièces fracturées et un produit directement lié à la fracturation de certains artefacts de faible épaisseur.

Cet éclat, appelé provisoirement «éclat de type SaintBrice» se caractérise par la présence d'un cône entièrement dégagé le plus souvent perpendiculairement à la face plane de l'éclat ou de la face inférieure du nucléus, voire de la pièce bifaciale (fig. 9).

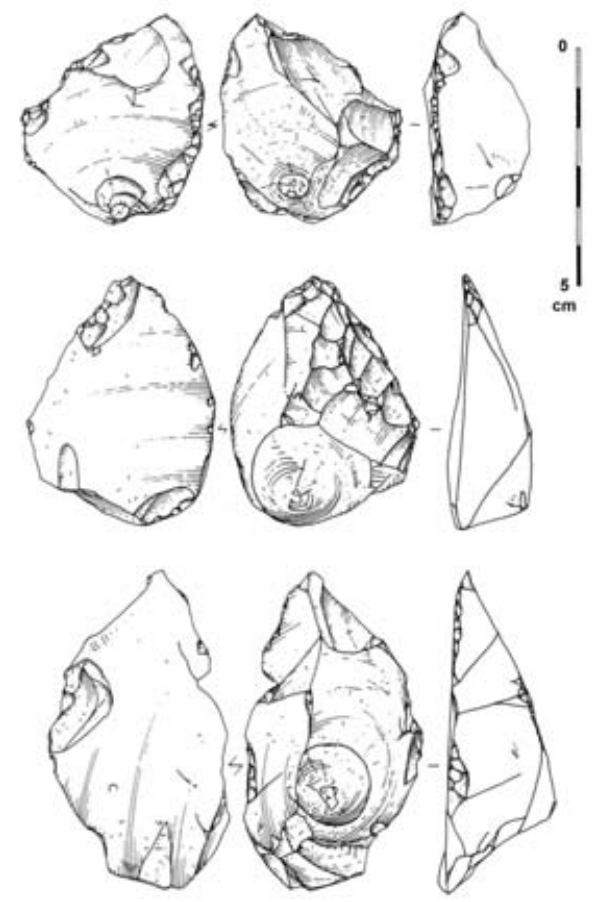

Fig. 9: Eclats de type Saint-Brice (dessin P. Alix). Fig. 9: Saint-Brice flake type (drawing P. Alix).
Le processus d'obtention de ces éclats a été reconnu expérimentalement (J. Ladjadj). Dans l'état actuel des investigations, ces éclats de type Saint-Brice pourraient être dus à la fracturation volontaire de pièces dans le but de réaménager deux surfaces de plan de frappe sur deux pièces obtenues depuis un seul support initial. Seuls quelques éléments de la chaîne opératoire ont été reconnus et la recherche de remontage dans le but de valider les observations faites lors de l'expérimentation s'est avérée décevante.

Les autre types de fractures s'inscrivent dans un répertoire plus classique, telles celles observées par Lhomme (2000) sur les sites de Champlost (Yonne) et "Chez-Pourré - Chez-Comte», à savoir: cassure par flexion, cassure par percussion et enfin cassure «indéterminée».

Les stigmates des deux premiers types de fracture ont été reconnus sur le mobilier de Saint-Brice, notamment sur des pièces retouchées (principalement racloirs à retouche biface et pièces bifaciales) présentant fréquemment des amincissements. Une expérimentation conduite sur des pièces obtenues sur le silex mis en œuvre par les paléolithiques de Saint-Brice devrait s'avérer éclairante, notamment quant à l'utilisation de certains outils (en cours).

Au terme de l'étude, il s'avère que la série n'est pas Levalloisienne et peu laminaire.

La lecture technologique des nucléus autorise quelques remarques (Bianchini, 2006). Le débitage direct, qui exploite les convexités naturelles des supports initiaux domine l'ensemble de la production. Les méthodes Kombéwa, Levallois, Discoïdes et Quina ont aussi été mises à profit par les Paléolithiques dans le but de produire des éclats "équilibrés» et relativement minces (débitage direct, «Clactonien», Levallois, Kombéwa, Quina, pièce recyclée) et des enlèvements plus trapus, dissymétriques, souvent larges et courts, arborant fréquemment un «dos» (débitage direct, Discoïde, Quina, pièce recyclée). Les recyclages d'ébauches et de pièces bifaciales achevées témoignent du changement de statut de certains objets au sein de l'assemblage (Boëda, 1997).

Les observations effectuées sur le mobilier attestent l'affûtage des racloirs et des pièces bifaciales. Il n'est pas impossible que le réaménagement des tranchants de certains racloirs à retouche bifaciale ait abouti à l'élaboration de pièces bifaciales. Des amincissements opposés aux «zones affûtées» ont été observés à plusieurs reprises. Ce constat irait dans le sens d'une évolution de la morphologie et parfois de l'utilisation de l'outil.

\subsection{3 - Diagnose}

Les assemblages lithiques à pièces bifaciales intègrent le grand complexe des industries à outils bifaciaux (fig. 10), notamment du Massif Armoricain et ses marges, principalement illustré, par les vastes sites d'ateliers, sur silex à La Girardière (Sarthe; Bourdin, 2006) et à Pierrepont (Calvados; Cliquet et al., 2003), sur grès éocènes au Bois du Rocher (Côtes d'Armor; Monnier, 


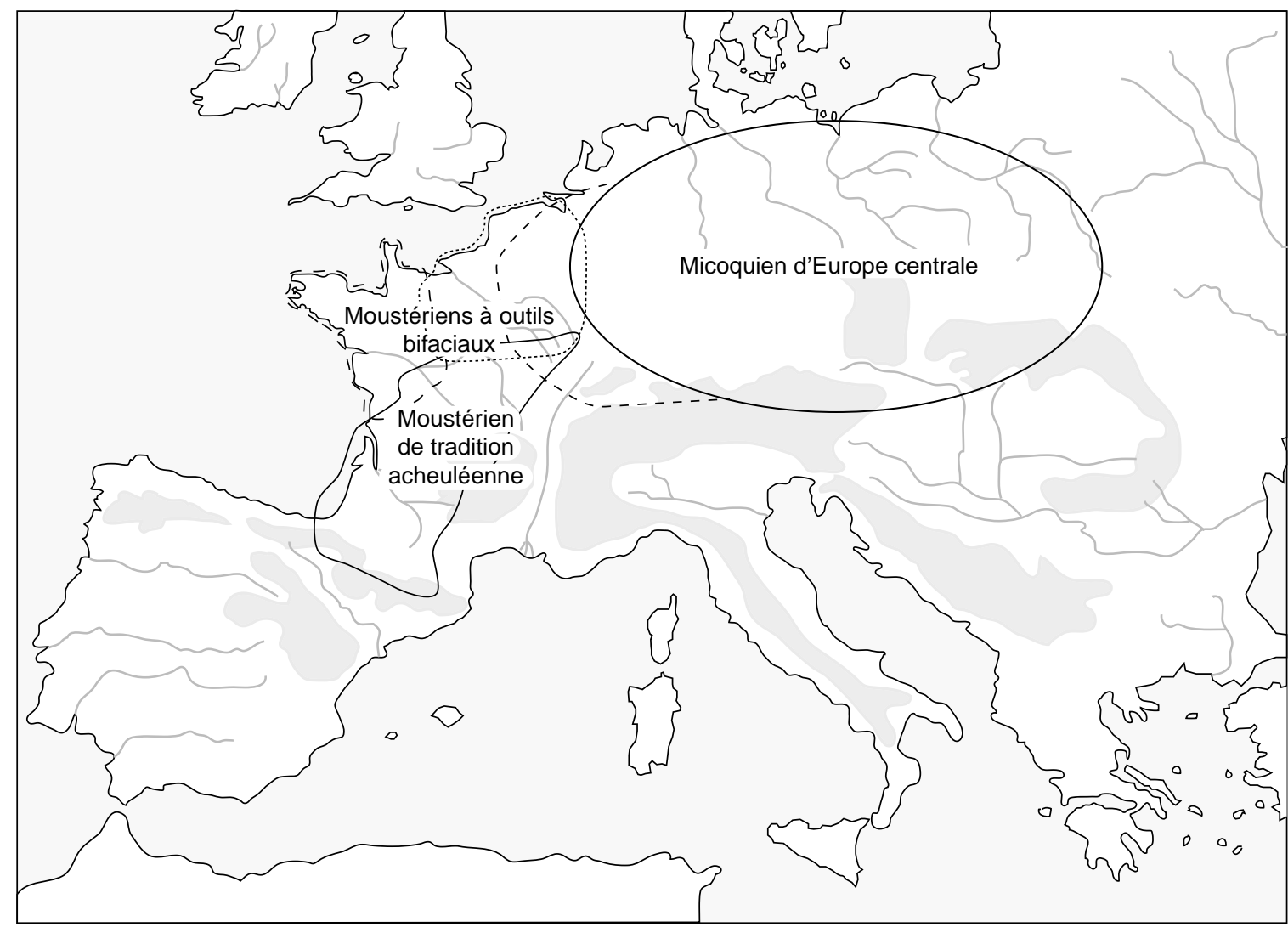

Fig. 10 : Localisation des industries bifaciales en Europe : MTA, Paléolithique moyen à outils bifaciaux et Micoquien (Europe moyenne et orientale) d'après les travaux de D. Cliquet et al., 2001, M. Soressi, 2002, S. Bourdin, 2006 \& J. Richter, 2006.

Fig. 10: Localisation of bifacial industries in Europe: MTA, Middle Palaeolithic with bifacial tools and the Micoquian (Central and Eastern Europe) from D. Cliquet et al., 2001, M. Soressi, 2002, S. Bourdon, 2006 \& J. Richet, 2006.

1980; Bourdin, 2006) et à Kervouster (Finistère; Monnier 1980; Bourdin, 2006), sur microgranite à Karreg-Ar-Yellan (Côtes d'Armor; Monnier, 1989; Huet, 2006)...

Ces ateliers de production d'outils bifaciaux se retrouvent dans la grande plaine lœssique, comme à Saint-Julien-de-la-Liègue (Eure; Cliquet, 1995; Pinoit, 2001), ou à Saint-Amand-les-Eaux (Nord; Deschodt et al., 2006).

Pour le domaine armoricain, seul le gisement de Saint-Brice-sous-Rânes est actuellement daté par les méthodes radiométriques (par TL sur silex chauffés; cf. infra).

\section{4 - DES NAPPES DE VESTIGES CONSTITUANT UN VASTE ATELIER DE PRODUCTION D'OUTILS BIFACIAUX}

Bien que nos investigations ne soient que partielles au regard de l'étendue de la surface d'atelier (plusieurs centaines d'hectares), l'analyse en cours a cependant apporté quelques éléments de réponses aux questions restées en suspens. Notamment, le fait que le sol d'occupation étudié soit relativement bien circonscrit dans l'espace.

Cette aire s'avère bien démarquée de celle qui a été reconnue en place environ $75 \mathrm{~m}$ au sud-ouest. Cependant, l'industrie issue du sondage P 231 - S 9 présente de grandes similitudes avec le mobilier collecté en P6 TR 1, bien que notre diagnostic ne repose que sur 61 pièces prélevées afin de ne pas affecter l'intégrité du site.

À la faveur de ces observations, le gisement pourrait être constitué d'une nébuleuse de nappes de vestiges bien circonscrites. L'impression d'un gigantesque palimpseste est vraisemblablement due aux remaniements liés aux diverses actions humaines: excavations destinées à l'extraction de minerai de fer, déstabilisation des sols favorisant l'érosion suite à l'arrachage des haies. Par ailleurs, cette apparence uniforme est accentuée par l'épandage des mobiliers lors des labours.

\section{1 - ANALYSE SPATIALE DU SITE FOUILLÉ: VERS UNE APPROCHE TAPHONOMIQUE}

Les résultats préliminaires de l'analyse spatiale ne permettent pas d'attribuer la nappe de vestiges à une seule occupation (l'analyse 3D des données est en cours). Il serait donc illusoire de tenter une interprétation de la surface d'occupation à ce stade de l'étude. Nous ne pouvons que constater certains faits illustrés par les raccords et remontages de pièces lithiques (fig. 11) et les plans de densimétries.

Les raccords et les remontages s'avèrent peu nombreux. Leur plan de répartition témoigne d'une certaine dispersion des artefacts constituant quelques ensembles. Néanmoins, plusieurs groupements sont attestés, notamment en G10 et C10 ; ils pourraient corres- 


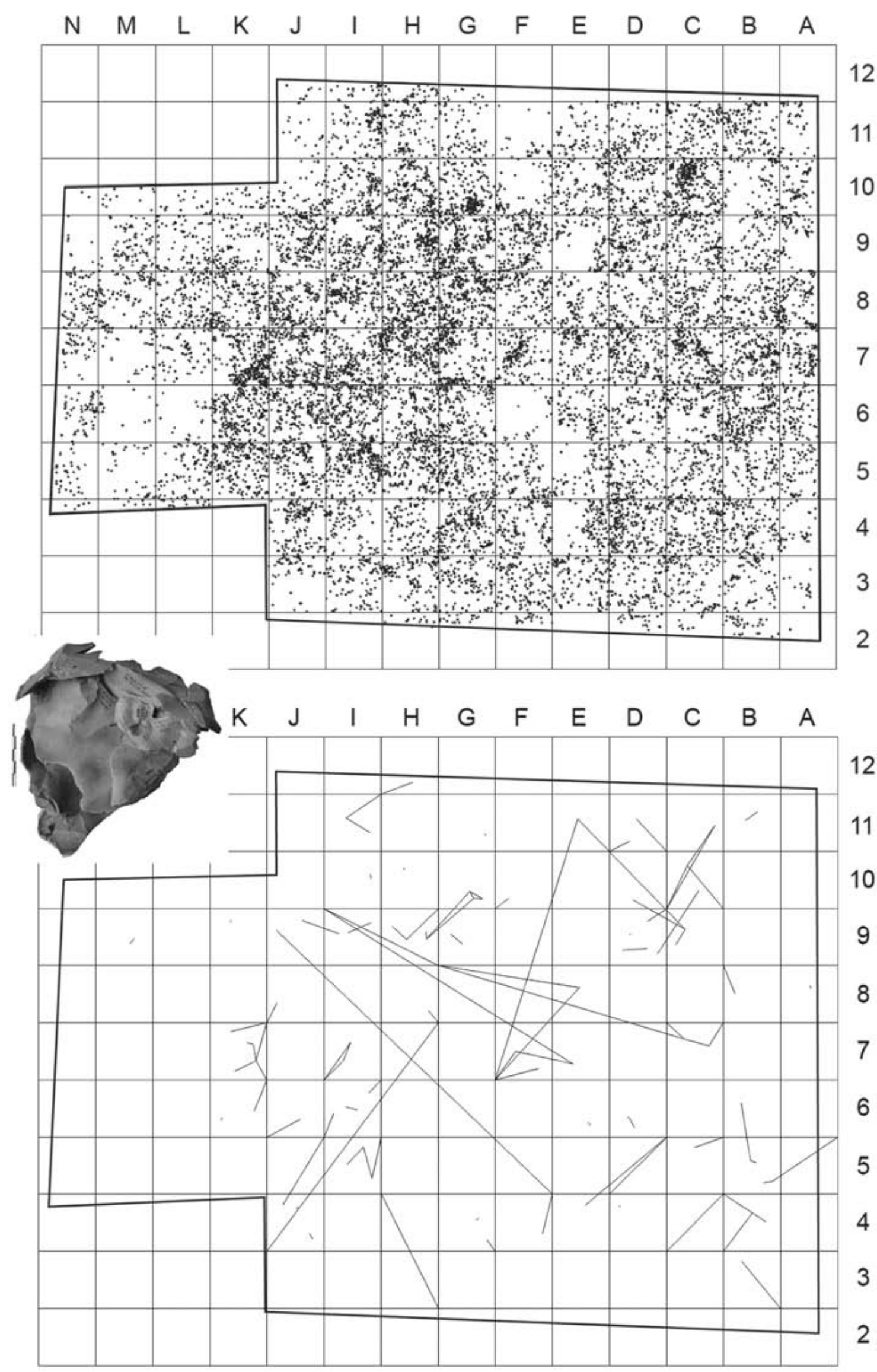

Fig. 11 : Répartition spatiale des artefacts, et des raccords et des remontages effectués.

Fig. 11: Spatial distribution of the artefact scatters, and the connexions and refitting achieved. 
pondre soit à des amas de débitage et / ou de façonnage, soit à des aires de rejet de produits de débitage.

L'analyse des données se poursuit afin de préciser la dynamique des éléments au sein du niveau archéologique (recours à la $3 \mathrm{D}$ ), de tenter de préciser si nous sommes en présence d'une nappe de vestiges correspondant à une seule occupation, ou plutôt à une succession d'installations sur le gîte de matière première (palimpseste), de mieux appréhender l'organisation spatiale de l'atelier, en intégrant les observations taphonomiques.

\section{2 - NATURE ET FONCTION DU SITE FOUILLÉ}

Les hypothèses initialement énoncées (Cliquet et al., 2001) semblent corroborées par les observations qui ont pu être effectuées postérieurement.

La présence d'amas de produits de débitage et de façonnage, d'outils (bifaciaux et «sur éclat»), réaménagés sur place (éclats de coup de tranchets, de retaille de biface, d'affûtage de pointe d'outils bifaciaux, de retouche) laisse supposer que l'espace ouvert du site participerait à un complexe atelier / aire d'activité(s). L'abondance des esquilles témoigne du caractère in situ de l'industrie.

Les pièces corticales restent en faible nombre. Ce fait pourrait correspondre, soit à une stratégie de division du travail de débitage et / ou de façonnage - l'épannelage des blocs aurait été effectué en un autre lieu -, soit à une adaptation liée à la disponibilité des matières premières les hommes préhistoriques ont utilisé des blocs gélivés -.

Cette économie dans la gestion de la matière d'œuvre semble attestée par l'entretien de l'outillage jusqu'à exhaustion complète, et par le recours aux roches locales: grès tertiaire, grès quartzite, quartz.

La densité de mobilier, entre 300 et 2000 pièces au $\mathrm{m}^{2}$, dans l'état actuel d'avancement des travaux, devrait permettre d'effectuer une étude taphonomique et spatiale fine de la couche d'occupation (les remontages d'artefacts lithiques sont jusqu'alors peu éclairants). S'agit-il d'une halte courte, d'une occupation longue, de la superposition de plusieurs implantations espacées dans le temps et ne formant plus qu'une seule couche (palimpseste)?

Comme nous l'avons précédemment évoqué, l'atelier de Saint-Brice-sous-Rânes évoque les grands gisements de production d'outils bifaciaux d'Armorique et du plateau de Madrie (entre Seine et Eure).

Les témoins de combustion y sont présents, bien que peu nombreux. Ils attestent de la proximité d'une structure de combustion: foyer domestique ou feu lié à une ou plusieurs activités spécifiques? Rappelons cependant que les éléments chauffés s'avèrent rarissimes en occupations ouvertes de plein air.

\section{5 - APPORT DES DATATIONS EFFECTUÉES SUR SILEX CHAUFFÉS}

Les datations tentées sur le site de Saint-Brice-sousRânes intègrent notre programme de constitution d'une trame chronologique, initialement fondée sur la seule chronostratigraphie, notamment pour les sites où le bilan sédimentaire est peu développé ou mal conservé. Ce cadre permet de préciser les corrélations possibles entre la Bretagne, la Normandie et le Nord de la France.

Compte tenu de l'ancienneté présumée des dépôts (>30 ka), les matériaux disponibles pour la réalisation de datations radiométriques sont peu diversifiés. Les plus favorables sont les silex chauffés dans les foyers préhistoriques qui sont potentiellement datables par la méthode de la Thermoluminescence (TL); celle-ci permet en effet de déterminer le temps écoulé depuis la chauffe préhistorique. Jusqu'à présent, cette méthode n'a pu être que peu employée pour des gisements du Nord-Ouest de la France, à l'exception de ceux de Menez-Dregan 1 (Mercier et al., 2004) ou du Rozel, Port-Racine, Grossoeuvre (Cliquet et al., 2003). En effet, peu de sites de plein air du Paléolithique moyen livrent des témoignages de combustion. Ainsi, à Saint-Brice, sur plus de 100000 artefacts, seules quelques pièces présentaient des stigmates liés au feu.

De plus, la méthode de la thermoluminescence nécessite des échantillons permettant un prélèvement central sur lequel seront faites les mesures, ceci afin d'éliminer les effets microdosimétriques qui sont des sources d'incertitude mal contrôlées. Les zones corticales s'avèrent aussi inaptes à ces analyses. Ces critères font donc qu'une partie des artefacts collectés à la fouille n'est pas exploitée.

La méthode de datation par TL repose sur le fait que les silex enregistrent, de manière fiable, les doses de radiations auxquelles ils sont continuellement soumis lors de leur enfouissement. Ils constituent ainsi d'excellents dosimètres qui sont réinitialisés dès que leur température excède $380{ }^{\circ} \mathrm{C}$; la chauffe archéologique constitue donc l'évènement daté puisqu'elle induit une remise à zéro du chronomètre en effaçant les doses préalablement reçues. La connaissance de la dose accumulée depuis la chauffe préhistorique (ou paléodose, notée également ED) et celle du débit de dose moyen reçu annuellement permettent alors, par division de ces deux facteurs, de déterminer l'âge TL.

Parmi les fragments de silex récoltés sur le gisement (fig. 12), six présentaient des marques indiquant une chauffe dans le passé et possédaient une masse suffisante pour les expériences. L'analyse préliminaire de leurs signaux de thermoluminescence a montré que ces fragments avaient en effet été soumis à des températures élevées, ayant conduit à l'effacement du signal TL exploité pour la datation, situé à $380^{\circ} \mathrm{C}$. Ces échantillons (SBR 1 à 6) ont alors été traités de manière à les dater par TL.

À cette fin, chaque échantillon a été scié, réduit en poudre et la fraction granulométrique 100-160 $\mu \mathrm{m}$ nettoyée à l'acide chlorhydrique (Valladas 1992). Une partie de la poudre obtenue a été divisée en quatre lots dont trois ont été irradiés avec des doses croissantes, délivrées par une source de Cs-137 (débit de dose gamma: $1.3 \mathrm{~Gy} / \mathrm{min}$ ), dans le but de les vieillir artificiellement. La mesure des signaux de la thermoluminescence cumulée - TL $(\mathrm{N}+\mathrm{A})$ - a ainsi permis de construire la courbe de croissance générale de la TL en fonction de la dose reçue. L'extrapolation de cette courbe pour une intensité TL nulle a fourni une estimation de la Dose 


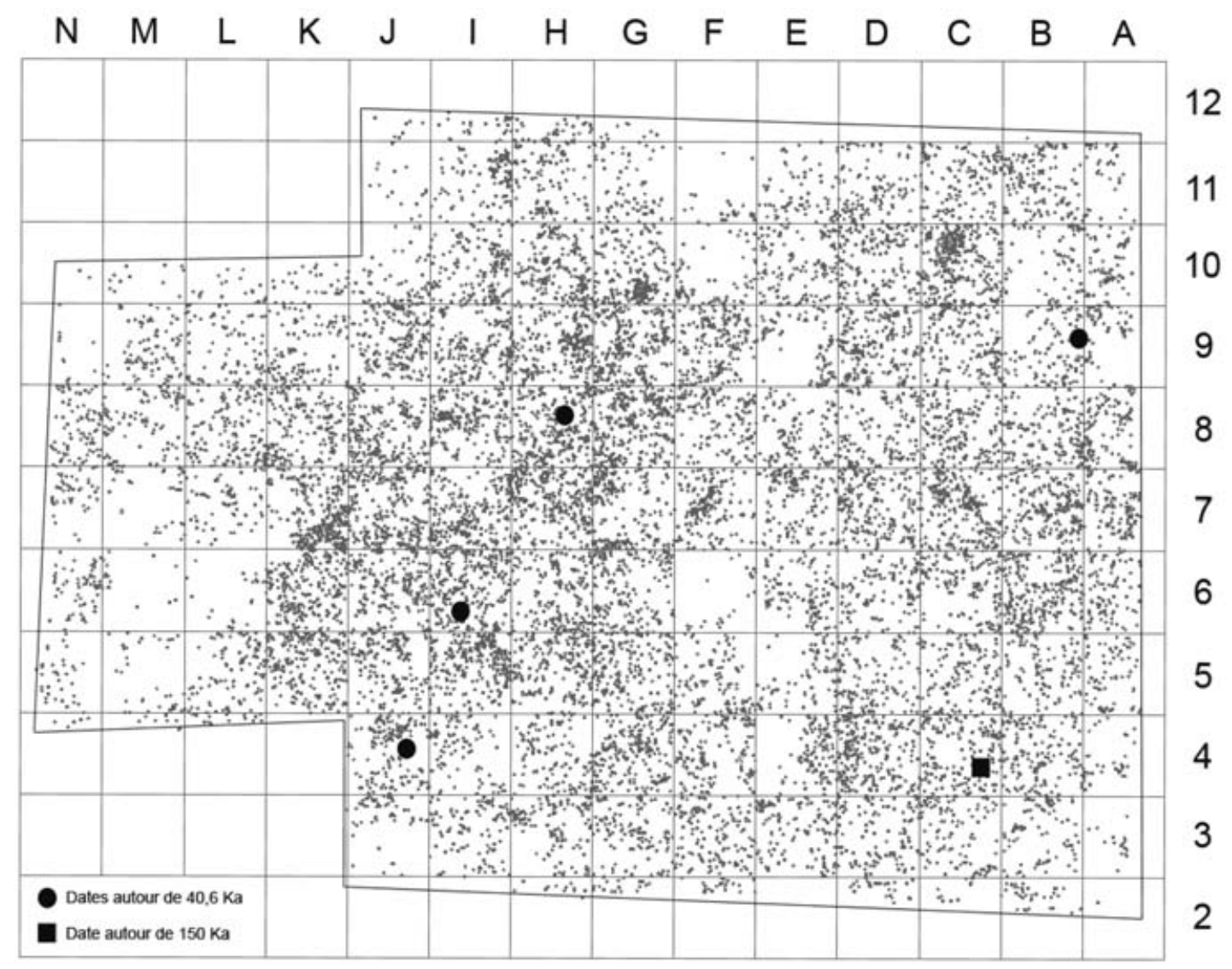

Fig. 12 : Localisation des silex chauffés sur le site d'atelier

Fig. 12: Localisation of the heated flints on the workshop site.

Equivalente (ED) mais celle-ci a dû être corrigée pour tenir compte des effets non-linéaires de croissance du signal TL (effet de supralinéarité) (Mercier et al., 1992). Pour déterminer cette correction, une seconde partie de la poudre originelle a été chauffée à $350{ }^{\circ} \mathrm{C}$ pendant 90 minutes de manière à effacer le signal de TL naturel, et divisée en quatre lots qui ont été irradiés en gamma à des doses croissantes. Les signaux induits ont alors été mesurés dans les mêmes conditions que précédemment. Les doses équivalentes déduites de ces analyses (tab. 2) sont voisines de l'ordre de 60-70 Gy pour cinq échantillons, alors que SBR3 présente une dose accumulée environ trois fois supérieure $(220 \pm 16 \mathrm{~Gy})$. Nous reviendrons sur cette observation lors de la discussion des âges.

Le débit de dose annuel a par ailleurs été mesuré en laboratoire et sur le terrain. Ce débit dépend des teneurs en radioéléments de l'échantillon (U, Th, K) qui indui- sent une dose dite interne puisqu'issue de l'échantillon lui-même, résultant de l'interaction des particules alpha et beta, de faibles parcours, avec la silice. Ces teneurs ont été mesurées par activation neutronique au Laboratoire Pierre Süe (Saclay) à partir de la poudre broyée. Elles sont voisines pour K (entre 0.02 et $0.04 \%$, sauf SBR5: $0.003 \%$ ), pour Th (entre 0.28 et $0.36 \mathrm{ppm}$ ) et pour U (entre 0.58 et $0.97 \mathrm{ppm}$, sauf SBR6: $1.92 \mathrm{ppm}$ ) (tab. 2). Les débits de dose internes sont donc assez voisins pour tous les échantillons (de 316 à $507 \mu \mathrm{Gy} / \mathrm{a}$ ) sauf SBR6 pour lequel il atteint $1032 \pm 98 \mu \mathrm{Gy} / \mathrm{a}$ du fait de la teneur élevée en Uranium de ce silex.

Quant à la dose dite externe, due au rayonnement gamma et dans une moindre mesure au rayonnement cosmique, elle a été calculée à partir de mesures réalisées sur le terrain à l'aide de dosimètres plantés dans les niveaux d'occupation où ils sont restés enfouis plusieurs mois, et a été trouvée remarquablement homogène.

\begin{tabular}{|c|c|c|c|c|c|c|c|c|c|c|c|c|c|c|}
\hline \multicolumn{2}{|c|}{ Echantillon } & \multirow{2}{*}{$\begin{array}{c}U \\
(\mathrm{ppm})\end{array}$} & \multirow{2}{*}{$\begin{array}{c}\text { Th } \\
\text { (ppm) }\end{array}$} & \multirow{2}{*}{$\begin{array}{c}\mathrm{K} \\
(\%)\end{array}$} & \multirow[b]{2}{*}{ Interne } & \multirow[b]{2}{*}{ - } & \multicolumn{3}{|c|}{ DOSES ( $\mu$ Gy/a) } & \multirow{2}{*}{$\stackrel{+}{-}$} & \multirow{2}{*}{$\begin{array}{l}\text { ED } \\
\text { (Gy) }\end{array}$} & \multirow{2}{*}{\multicolumn{2}{|c|}{$\begin{array}{c}\text { Age } \\
\text { (kans) }\end{array}$}} & \multirow{2}{*}{+} \\
\hline$n^{\circ}$ & Carré & & & & & & Externe & 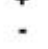 & Annuelle & & & & & \\
\hline SBR1 & $\mathrm{H} 8$ & 0.81 & 0.31 & 0.02 & 341 & 22 & 1216 & 48 & 1558 & 53 & 65.1 & 5.1 & 41.8 & 3.7 \\
\hline SBR2 & B9 & 0.78 & 0.28 & 0.04 & 414 & 29 & 1119 & 49 & 1533 & 57 & 65.3 & 5 & 42.6 & 3.6 \\
\hline SBR3 & $\mathrm{C} 4$ & 0.58 & 0.35 & 0.04 & 316 & 21 & 1222 & 49 & 1538 & 53 & 220.0 & 16 & 143.0 & 12.0 \\
\hline SBR4 & 16 & 0.76 & 0.31 & 0.03 & 366 & 27 & 1206 & 48 & 1571 & 55 & 65.6 & 4.9 & 41.8 & 3.6 \\
\hline SBR5 & $\mathrm{J} 4$ & 0.97 & 0.29 & 0.00 & 507 & 39 & 1195 & 47 & 1702 & 61 & 69.7 & 5.6 & 41.0 & 3.5 \\
\hline SBR6 & 16 & 1.92 & 0.36 & 0.03 & 1032 & 98 & 1184 & 47 & 2215 & 109 & 84.4 & 2.2 & 38.1 & 3.0 \\
\hline
\end{tabular}

Tab. 2 : Données radiométriques et âges TL des silex chauffés.

Tab. 2: Radiometric data and TL dating of burnt flints. 
Les âges TL obtenus (tab. 2) sont voisins, pour cinq d'entre eux, de 41 ka ce qui conforte l'idée que ces échantillons proviennent d'une nappe de vestiges qui, à la fouille, semble homogène. Cependant, au regard de l'extrême densité des artefacts (plus de 1000 pièces au $\mathrm{m}^{2}$ ) et des processus de dessiccation qui ont affecté le sol archéologique, il n'est pas exclu que nous soyons en présence d'un palimpseste de sols. La poursuite de l'analyse spatiale 3D des artefacts apportera quelques éclaircissements.

Cependant, un échantillon (SBR3) donne, du fait de sa dose équivalente élevée, une date $143 \pm 12 \mathrm{ka}$ (C4). Il se pourrait donc que cette pièce soit rapportable aux lambeaux d'occupation plus ancienne intégrée au cailloutis (série blanche?) (cf. supra).

\section{1 - SAINT-BRICE-SOUS-RÂNES PAR RAPPORT AU CADRE CHRONOLOGIQUE EUROPÉEN FONDÉ SUR LES DATATIONS ABSOLUES}

Les dates TL obtenues rapporteraient l'occupation de Saint-Brice-sous-Rânes à la période de transition entre le Paléolithique moyen et le Paléolithique supérieur, soit à la «charnière» de deux mondes, au plan humain et au plan conceptuel (Cliquet et al., 2005). De nombreux autres gisements sont associés, grâce aux datations radiométriques, à cette tranche temporelle et un certain nombre est porté dans les tableaux 3 et 4 .

L'ensemble de ces données évoque donc des gisements contemporains de l'occupation de Saint-Brice qui, de fait, peut être mise en perspective par rapport aux derniers peuplements rapportables au Moustérien et surtout aux premières incursions des Hommes modernes.

Dans cet intervalle temporel, nombre de cultures ont été identifiées (Djindjian et al., 1999; Gamble, 1999); ainsi, on y retrouve:

\section{* au Paléolithique moyen final}

- principalement le Moustérien de tradition Acheuléenne du sud-ouest de la France (Soressi, 2002) et du nord de la Péninsule ibérique, daté entre 60 et $35 \mathrm{ka}$;

\begin{tabular}{|c|c|c|}
\hline \multicolumn{3}{|c|}{ Jerzmaniwicien } \\
\hline \multicolumn{3}{|c|}{ Nietoperzowa (Jerzmanowice, Cracovie, Pologne) } \\
\hline 6 & $385001240 \mathrm{BP}$ & GrN2 181 \\
\hline \multicolumn{3}{|c|}{ Kent's cavern (Lincombe, Devonshire, Angleterre) } \\
\hline & $38270+1470 /-1240 \mathrm{BP}$ & GrN 6324 \\
\hline \multicolumn{3}{|c|}{ Paléolithique supérieur indẻterminé } \\
\hline \multicolumn{3}{|c|}{ Willendorf II (Basse-Autriche) } \\
\hline 2-D1 som & $39500+1500 /-1200 \mathrm{BP}$ & GrN 11190 \\
\hline \multicolumn{3}{|l|}{ Bohunicien } \\
\hline \multirow{2}{*}{\multicolumn{3}{|c|}{ Abris de Stranska Skalla III (Brno, Moravie) }} \\
\hline & & GrN 12297 \\
\hline Szélétien & & \\
\hline \multicolumn{2}{|c|}{ Szeleta (Borsod, M ontagnes de Bukk, Hongrie) } & \\
\hline inf. & $430001100 \mathrm{BP}$ & GrN6 058 \\
\hline
\end{tabular}

Tab. 3 : Sélection de dates ${ }^{14} \mathrm{C}$ des industries de transition (d'après Djindjian et al., 1999). Les références encadrées intègrent la fourchette chronologique de l'occupation du site de Saint-Brice.

Tab. 3: Selection of ${ }^{14} \mathrm{C}$ dates for the transition industries (from Djindjian et al., 1999). References between brackets take the chronological range of the occupation of the Saint-Brice site into account.
- le Moustérien final, du centre-sud de la Péninsule ibérique et du sud de la France, daté entre 60 et 28 ka;

\section{* au Paléolithique supérieur initial}

- le Bohunicien, d'Europe moyenne-orientale, daté entre 44 et $38 \mathrm{ka}$. Ce faciès culturel se caractérise par une production Levallois relativement bien exprimée (IL: 10), moins cependant que le débitage laminaire. À cette culture se rapportent notamment les vastes ateliers de débitage de silex jurassique de Stranska Skala. Les pièces foliacées et les racloirs typiques apparaissent façonnés sur d'autres matières premières.

- le Szélétien, d'Europe moyenne-orientale et centrale, daté entre 43 et $35 \mathrm{ka}$. La phase ancienne du Szélétien se caractérise par une «technologie moustérienne» à éclats, la production laminaire restant faible. L'outillage est à base de racloirs, de pointes foliacées, mais comporte encore de nombreux bifaces micoquiens, foliacés (faustkeilblatt) et à dos (keilmesser) qui traduiraient une origine rapportable au Micoquien d'Europe Centrale.

- le Bachokirien ou Proto-Aurignacien d'Europe centrale, daté de 43 ka environ.

- l'Aurignacien ancien, du centre-sud de la Péninsule ibérique et du sud de la France, daté entre 43 et $33 \mathrm{ka}$. C'est la première culture du Paléolithique supérieur attribuable à l'Homme moderne.

- l'Uluzzien d'Italie, daté de manière fort imprécise de plus de $33 \mathrm{ka}$.

- le Châtelperronien, du centre-sud de la Péninsule ibérique et du sud de la France, daté entre 37 et 33 ka.

\section{* aux assemblages à pointes foliacées}

- l'Altmühlien, d'Europe moyenne-orientale, daté d'environ $38 \mathrm{ka}$. Ce faciès culturel du Paléolithique moyen final se caractérise par le façonnage de pièces foliacées bifaciales (blattspitzen), de racloirs dont certains bifaciaux et de lames. Le débitage Levallois est attesté. Comme pour le Szélétien ancien, l'origine de l'Altmühlien est rapportée au Micoquien d'Europe Centrale.

- le complexe Lincombien / Ranisien / Jerzmanovicien, d'Europe du Nord-ouest et d'Europe moyenne, daté entre 39 et $36 \mathrm{ka}$.

La phase ancienne des assemblages rapportables à ce complexe se rattache aux industries à pointes foliacées du Paléolithique moyen de Grande-Bretagne, d'Allemagne et de Pologne (Otte, 1996; Richter, 2004).

Un des points essentiels caractérisant cette transition Paléolithique moyen / Paléolithique supérieur est la «floraison» des cultures à pièces bifaciales, notamment dites à «pointes foliacées». L'examen des planches proposées dans les publications et l'observation de quelques séries fait apparaître une utilisation fréquente du mot pointe foliacée pour des pièces bifaciales qui seraient, presque systématiquement attribuées aux bifaces dans la littérature française (cf. Cliquet, 1982, 1995; Cliquet \& Lautridou, 1988) (fig. 13 et 14).

En somme, dans les grandes plaines du nord, de l'Angleterre à la Pologne, les industries à pointes folia- 


\begin{tabular}{|c|c|c|c|c|c|}
\hline Site & Niveau & $\begin{array}{l}\text { Méthode de } \\
\text { datation }\end{array}$ & \multicolumn{2}{|c|}{ Culture } & \multirow{2}{*}{$\begin{array}{c}\text { Date kans } \\
\text { BP }\end{array}$} \\
\hline El Castillo & $18 \mathrm{~b} 2$ & ${ }^{14} \mathrm{C}$ & Paléolithique supérieur initial & Aurignacien & \\
\hline L'Arbreda & E2BE 111-1 & ${ }^{14} \mathrm{C}$ (AMS) & Paléolithique supérieur initial & Aurignacien & $37,7 \pm 1$ \\
\hline L'Arbreda & E2BE 111-2 & ${ }^{14} \mathrm{C}$ (AMS) & Paléolithique supérieur initial & Aurignacien & $37,7 \pm 1$ \\
\hline Gato Preto & Date moyenne & $\mathrm{TL}$ & Paléolithique supérieur initial & Aurignacien & $38,1 \pm 3$ \\
\hline Saint-Césaire & Niveau 11, Egp & $\mathrm{TL}$ & Paléolithique moyen & Moustérien à denticulés & $38,2 \pm 3,3$ \\
\hline Stranska Skala III & 5 & ${ }^{14} \mathrm{C}$ & Paléolithique supérieur initial & Bohunicien & $38,2 \pm 1,1$ \\
\hline Kents Cavern & Niveau A2 & ${ }^{14} \mathrm{C}$ & Paléolithique supérieur initial & Lincombien & $38,2 \pm 1,4$ \\
\hline Certova Pec & & ${ }^{14} \mathrm{C}$ & Paléolithique supérieur initial & Szélétien & $38,4 \pm 2,8$ \\
\hline Stranska Skala III & 5 & ${ }^{14} \mathrm{C}$ & Paléolithique supérieur initial & Bohunicien & $38,5 \pm 1,4$ \\
\hline El Castillo & $18 \mathrm{~b} 1$ & ${ }^{14} \mathrm{C}$ (AMS) & Paléolithique supérieur initial & Aurignacien & $38,5 \pm 1,8$ \\
\hline Korolevo 2 & 2 & ${ }^{14} \mathrm{C}$ & Paléolithique supérieur initial & non caractérisé & $38,5 \pm 1$ \\
\hline Nietoperzowa & couche 6 & ${ }^{14} \mathrm{C}$ & Paléolithique supérieur initial & Pointes foliacées & $38,5 \pm 1,2$ \\
\hline Grotte de Sclayn & niveau $1 \mathrm{~A}$ & ${ }^{14} \mathrm{C}$ & Paléolithique moyen & Moustérien & $38,6 \pm 1,5$ \\
\hline Külna & $7 a$ & ${ }^{14} \mathrm{C}$ & Paléolithique moyen & Micoquien & $38,6 \pm 0,95$ \\
\hline L'Arbreda & E2BE 111-4 & ${ }^{14} \mathrm{C}$ (AMS) & Paléolithique supérieur initial & Aurignacien & $38,7 \pm 1,2$ \\
\hline Temnata & TDII, niveau 6 & ${ }^{14} \mathrm{C}$ & Paléolithique supérieur initial & non caractérisé & $>38,7$ \\
\hline Douara & IV B supérieur & ${ }^{14} \mathrm{C}$ & Paléolithique moyen & Moustérien & 38 \\
\hline Combe-Grenal & 12 & ${ }^{14} \mathrm{C}$ & Paléolithique moyen & Moustérien à denticulés & $39 \pm 1,5$ \\
\hline L'Arbreda & E2BE 116-1 & ${ }^{14} \mathrm{C}$ (AMS) & Paléolithique moyen & Moustérien & $39,4 \pm 1,4$ \\
\hline Coudoulous II & stalagmite du sol 2 & $\mathrm{U} / \mathrm{Th}$ & Paléolithique moyen & Moustérien & $39,4 \pm 0,5$ \\
\hline Willendorf II & 2 & ${ }^{14} \mathrm{C}$ & Paléolithique supérieur initial & Aurignacien & $39,5 \pm 1,5$ \\
\hline Vedrovice $\mathrm{V}$ & & ${ }^{14} \mathrm{C}$ & Paléolithique supérieur initial & Szélétien & $39,5 \pm 1,1$ \\
\hline Istallosko & & ${ }^{14} \mathrm{C}$ & Paléolithique supérieur initial & Aurignacien & $39,7 \pm 09$ \\
\hline Cotencher & couche V & ${ }^{14} \mathrm{C}$ & Paléolithique moyen & Moustérien & $39,7 \pm 1,2$ \\
\hline L'Arbreda & E2BE 111-3 & ${ }^{14} \mathrm{C}$ (AMS) & Paléolithique supérieur initial & Aurignacien & $39,9 \pm 1,3$ \\
\hline Abric Romani & & «U-series \# & Paléolithique moyen & Moustérien & $39-60$ \\
\hline El Castillo & $18 \mathrm{c}$ & ${ }^{14} \mathrm{C}$ (AMS) & Paléolithique supérieur initial & Aurignacien & $40 \pm 2,1$ \\
\hline Riparo-Fumane & A2? & ${ }^{14} \mathrm{C}$ & Paléolithique supérieur initial & Aurignacien & $40 \pm 0,4$ \\
\hline Bohunice & 4 & ${ }^{14} \mathrm{C}$ & Indéterminé & Indéterminé & $40,1 \pm 1,2$ \\
\hline Ripiceni-Izvor & IVN (âge minimum) & ${ }^{14} \mathrm{C}$ & Paléolithique moyen & Moustérien & $40,2 \pm 1,1$ \\
\hline Le Moustier & $\mathrm{J}$ & $\mathrm{TL}$ & Paléolithique moyen & Moustérien typique & $40,3 \pm 2,6$ \\
\hline Cotencher & couche V & ${ }^{14} \mathrm{C}$ & Paléolithique moyen & Moustérien & $40,9 \pm 1,1$ \\
\hline Le Moustier & 1 & $\mathrm{TL}$ & Indéterminé & Indéterminé & $40,9 \pm 5$ \\
\hline Saint-Césaire & niveau 10 , Egf & $\mathrm{TL}$ & Paléolithique moyen & Moustérien à denticulés & $40,9 \pm 2,5$ \\
\hline Stranska Skala IIla & 4 & ${ }^{14} \mathrm{C}$ & Paléolithique supérieur initial & Bohunicien & $41,3 \pm 3,1$ \\
\hline L'Arbreda & E2BE 116-3 & ${ }^{14} \mathrm{C}$ (AMS) & Paléolithique moyen & Moustérien & $41,4 \pm 1,6$ \\
\hline Bohunice & 4 & ${ }^{14} \mathrm{C}$ & Paléolithique supérieur initial & Bohunicien & $41,4 \pm 1,4$ \\
\hline Willendorf II & 2 & ${ }^{14} \mathrm{C}$ & Paléolithique supérieur initial & Aurignacien & $41,7 \pm 3,7$ \\
\hline Szeleta & $B$ & ${ }^{14} \mathrm{C}$ & Paléolithique supérieur initial & Szélétien & $>41,7$ \\
\hline $\begin{array}{l}\text { Frechet Gr de } \\
\text { Peyrère I }\end{array}$ & Niveau supérieur & ${ }^{14} \mathrm{C}$ & Paléolithique moyen & Moustérien & $42 \pm 3,1$ \\
\hline Saint-Césaire & Niveau 12, Egf & $\mathrm{TL}$ & Paléolithique moyen & Moustérien à denticulés & $42,4 \pm 4,8$ \\
\hline Ripiceni-Izvor & IV (âge minimum) & ${ }^{14} \mathrm{C}$ & Paléolithique moyen & Moustérien & $42,5 \pm 1,3$ \\
\hline Le Moustier & $\mathrm{H} 2-\mathrm{H} 9$ & $\mathrm{TL}$ & Paléolithique moyen & Moustérien de Trad. Acheul. B & $42,5 \pm 2$ \\
\hline Le Moustier & $\mathrm{K}$ & $\mathrm{TL}$ & Paléolithique moyen & Moustérien & $42,6 \pm 1,7$ \\
\hline Bohunice & 4 & ${ }^{14} \mathrm{C}$ & Paléolithique supérieur initial & Bohunicien & $42,9 \pm 1,7$ \\
\hline La Quina & $6 a$ & $\mathrm{TL}$ & Paléolithique moyen & Moustérien type Quina & $43 \pm 3,6$ \\
\hline Sant'Agostino & Niveau 1 & ESR & Paléolithique moyen & Pontinien & $43 \pm 9$ \\
\hline Bacho Kiro & couche 11 & ${ }^{14} \mathrm{C}$ & Paléolithique supérieur initial & & $>43$ \\
\hline Cotencher & Couche 6 & ${ }^{14} \mathrm{C}$ & Paléolithique moyen & Moustérien & $43,2 \pm 1$ \\
\hline Ripiceni-Izvor & IV (âge minimum) & ${ }^{14} \mathrm{C}$ & Paléolithique moyen & Moustérien & $43,8 \pm 1,1$ \\
\hline Königsaue & lb (âge minimum) & ${ }^{14} \mathrm{C}$ (AMS) & Paléolithique moyen & Micoquien & $43,8 \pm 2,1$ \\
\hline
\end{tabular}

Tab. 4 : Tableau des sites datés susceptibles d'être contemporains de l'occupation de Saint-Brice-sous-Rânes (d'après Gamble, 1999). Tab. 4: Table of dated sites that could be contemporary to the occupation of Saint-Brice-sous-Ranes (from Gamble, 1999). 


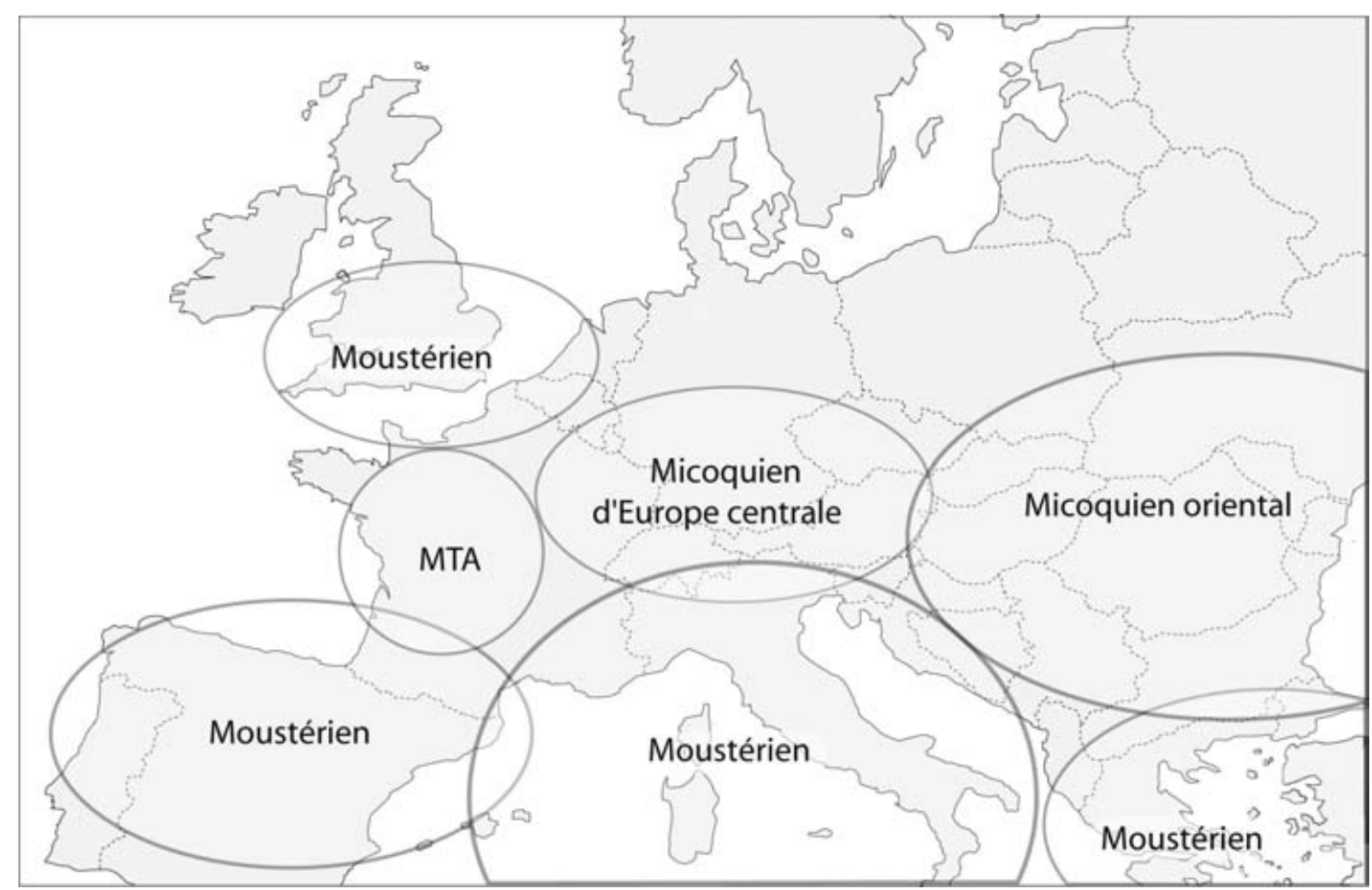

Fig. 13 : Traditions culturelles de la fin du Paléolithique moyen, en Europe (d'après Richter, 2006).

Fig. 13: Cultural traditions at the end of the Middle Palaeolithic in Europe (from Richter, 2006).

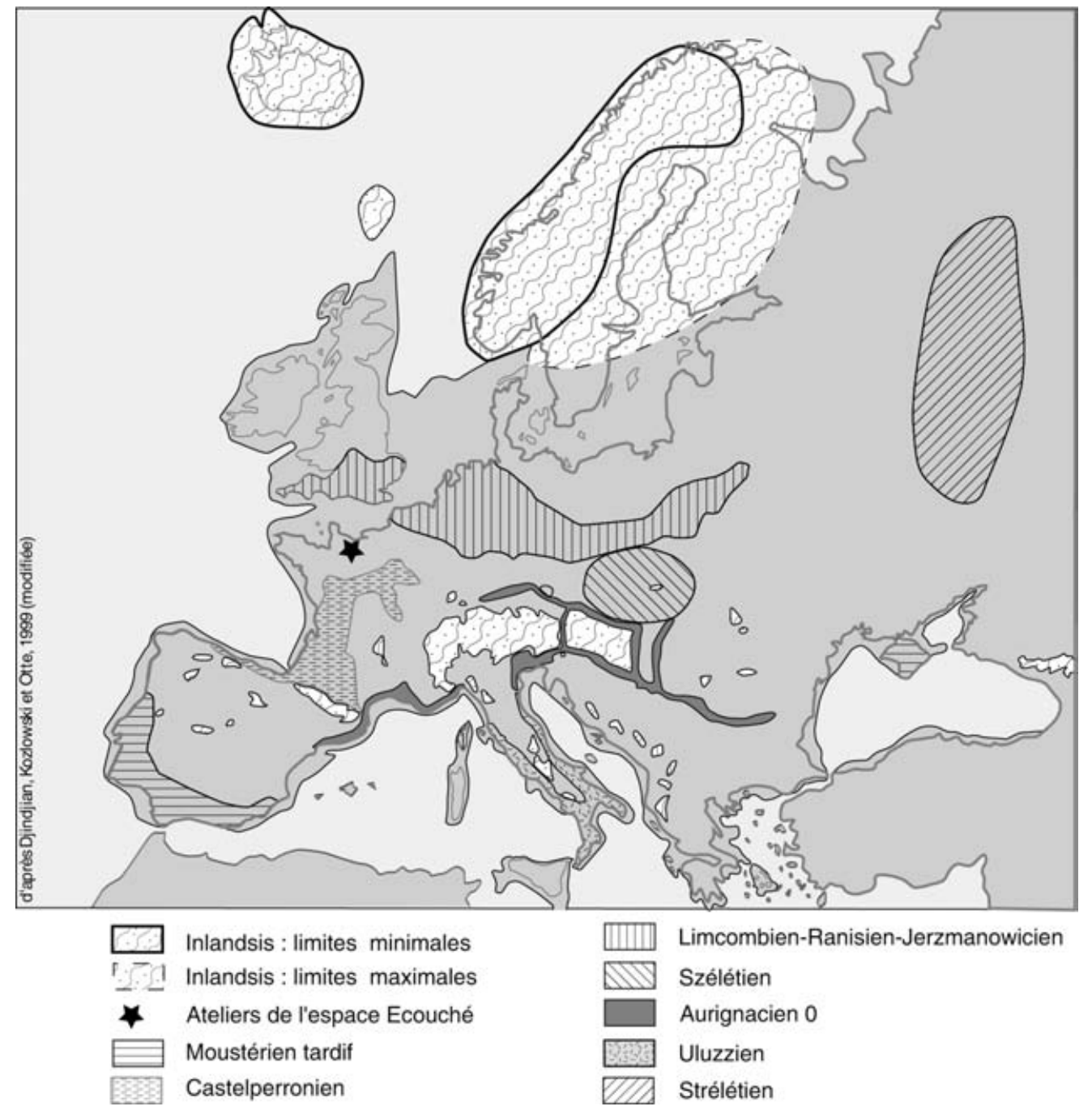

Fig. 14 : Extension des groupes culturels de la transition Paléolithique moyen / Paléolithique supérieur en Europe.

Fig. 14: Extension of the cultural groups at the Middle / Upper Palaeolithic transition in Europe. 
cées du Paléolithique moyen final se développent et s'étendent spatialement. Selon Otte (1996), «l'impression générale laissée par ces ensembles moustériens très évolués est qu'il s'agit d'évolutions autonomes vers la technologie paléolithique nouvelle», liée à l'extension de l'aire culturelle des premiers Hommes modernes. En effet durant l'interpléniglaciaire, les supports de l'outillage prennent une tendance laminaire, et les outils intègrent la panoplie instrumentale des artisans du Paléolithique supérieur: burins, grattoirs, troncatures...

Ce même phénomène se retrouve, plus vers l'Est, où des «industries aux pointes triangulaires ou foliacées bifaces semblent manifester les mêmes tendances évolutives. Le site de Sungir a donné son nom à ce faciès moustérien évolué du Bassin du Don (sites de Kostienki), du Pruth et du Dniestr (Moldavie et Ukraine)» (Otte, 1996).

Les assemblages normands de Saint-Brice-sousRânes (Orne), et vraisemblablement de Saint-Julien-dela-Liègue (Eure) pourraient figurer, à notre sens, parmi les cultures à pièces bifaciales de la transition.

Ce phénomène s'inscrit en plein Pléniglaciaire moyen (55 - $30 \mathrm{ka})$, soit autour de $40 \mathrm{ka}$. Ceci correspond à une période où la sédimentation lœssique est fortement réduite et interrompue par plusieurs phases de pédogenèse de type sol brun boréal à sol brun arctique, souvent représentées par un horizon unique polyphasé (Complexe de Saint-Acheul-Villiers-Adam) (Antoine \& Lautridou, 2003).

Pour le «créneau temporel» concerné par les derniers peuplements du site de Saint-Brice, les données environnementales attestent pour cette période de transition culturelle d'un épisode d'amélioration climatique (interstade de Moershoofd - entre 45 et 40 ka B.P.) (fig. 15). Bien que cet événement demeure mal documenté en Europe occidentale, faute, d'enregistrements polliniques, il en est tout autrement en-Europe orientale où les gisements ont livré des spectres polliniques qui s'avèrent plus explicites (vallées du Dniestr et du Don, Pologne et nord de la Russie) (Djindjian et al., 1999). Ces derniers témoignent pour les sites les plus occidentaux d'une végétation ouverte avec des agrégats d'arbres (Pinus, Larix, Pinus cembra), en d'autres termes une prairie à conifères.

L'environnement des néandertaliens de Saint-Brice devait présenter de grandes similitudes avec ces paysages plus orientaux. Le «pouvoir tampon» de l'océan jouait

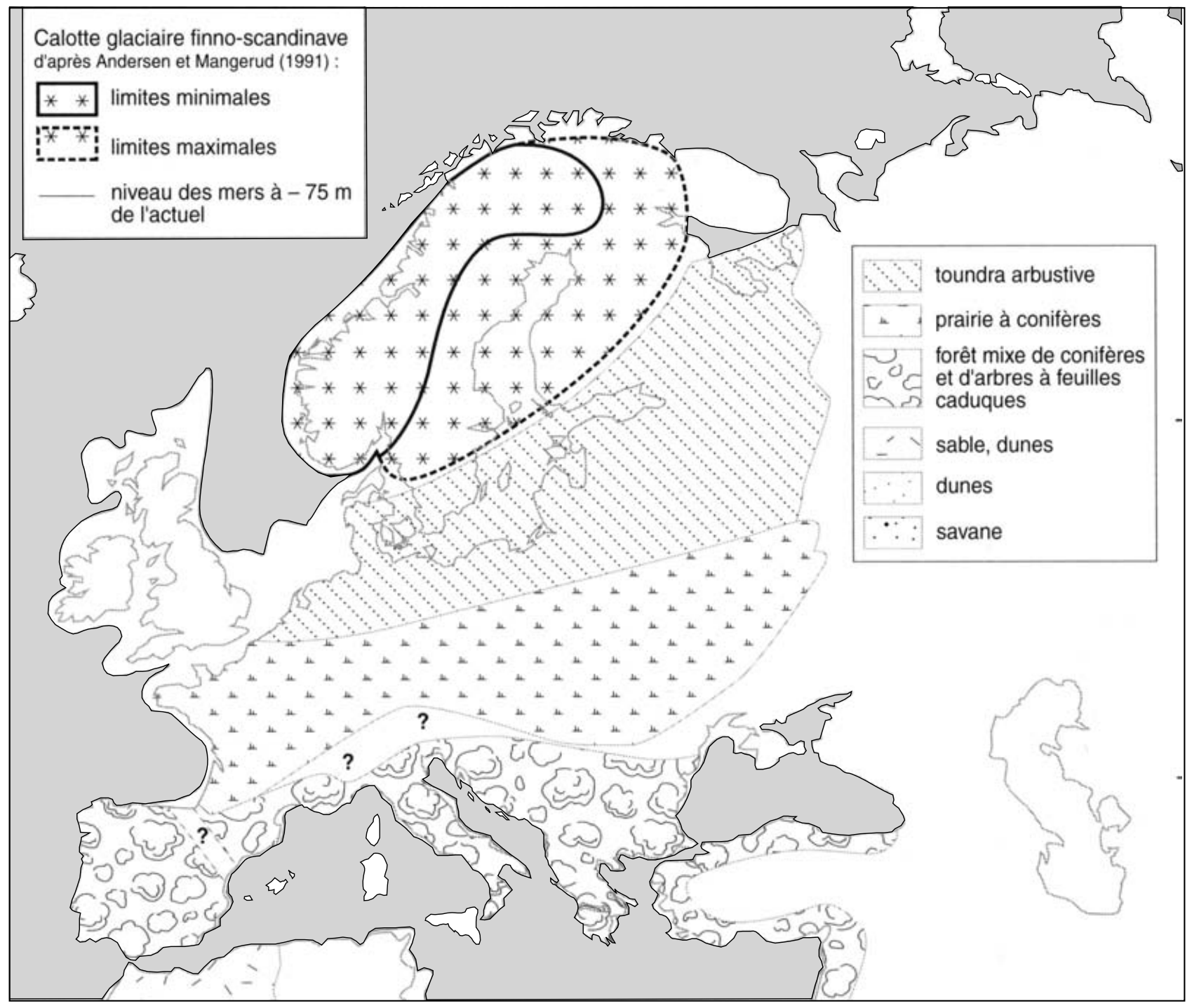

Fig. 15 : Carte de la végétation en Europe pendant l'interpléniglaciaire (d'après Van Andel \& Tzedakis, 1996). Fig. 15: Map of the vegetation in Europe during the interpleniglacial (from Van Andel \& Tzedakis, 1996). 
en effet un rôle beaucoup plus modéré qu'en période interglaciaire (influence du Gulf Stream) du fait de la transgression, estimée à $75 \mathrm{~m}$ sous le niveau marin actuel.

\section{6 - CONCLUSION ET PERSPECTIVES}

L'intérêt du site de Saint-Brice-sous-Rânes réside dans le fait que pour la première fois dans le Massif armoricain normand, une série lithique attribuée au Paléolithique moyen à outils bifaciaux a été collectée en place. Par ailleurs, la dernière occupation de l'espace fouillé est datée par TL sur silex chauffés de 40,6 $\pm 2,2 \mathrm{ka}$, soit de la période de transition entre le Paléolithique moyen et le Paléolithique supérieur. Rappelons qu'actuellement, le site de Saint-Brice-sous-Rânes est le seul atelier de production d'outils bifaciaux de l'Ouest de l'hexagone pour lequel nous disposons de datations radiométriques.

L'aire observée présente les caractéristiques d'un site d'atelier et de consommation sur lequel les outils étaient utilisés (configuration de site d'atelier et d'habitat?). Ce type d'occupation apparaît très original en Normandie hormis peut-être les stations de Saint-Julien-de-laLiègue dans le département de l'Eure.

Enfin, l'analyse (en cours) des «structures tangibles et latentes» du site notamment par la technique des remontages laisse présager une organisation de l'atelier (amas de débitage et de pièces de petites dimensions, répartition spatiale des nucléus, des pièces bifaciales, des outils et des artefacts fragmentés volontairement ou lors de leur utilisation) donc une lecture technologique de ce sol d'occupation.

\section{RÉFÉRENCES BIBLIOGRAPHIQUES}

ANDEL Th. (Van), \& TZEDAKIS C., 1996 - European Palaeolithics Landscapes: 140000- 30000 Years ago. A summary. In O. Bar Yosef, L.L. Cavalli-Sforza, R.J. March \& M. Piperno (eds), The origin of modern man. XIII ${ }^{\text {th }}$ UISPP Congress, Colloquium X, vol. 5, Forli, ABACO, 191-198.

ANTOINE P., ROUSSEAU D.-D., LAUTRIDOU J.-P., \& HATTÉ C., 1999 - Last interglacial-glacial climatic cycle in loess-palaeosol successions of north-western France. Boreas, 28, 551-563.

ANTOINE P., \& LAUTRIDOU J.-P., 2003 - La séquence du dernier cycle (Eemien - Weichselien) dans les loss de la France septentrionale. Pré-print du colloque international de Rennes, 2003, Les premiers peuplements en Europe, 20-21.

BIANCHINI R., 2006 - L'analyse technologique des nucléus du Paléolithique moyen final de Saint-Brice-sous-Rânes (Orne): détermination de la chaîne opératoire de débitage et intégration dans l'étude $d u$ site. Mémoire de Maîtrise, Université de Paris I, 66 p.

BOEDA E., 1997 - Technogénèse de systèmes de production lithique au Paléolithique inférieur et moyen en Europe occidentale et au Proche-Orient. Mémoire d'Habilitation à Diriger des Recherches, Université de Paris X/Nanterre, 173 p.

BORDES F., 1961 - Typologie du Paléolithique ancien et moyen. Bordeaux, Delmas, $85 \mathrm{p}$.

BOURDIN S., 2006 - Le Moustérien à outils bifaciaux du Massif armoricain au Pléistocène récent dans son contexte européen : vers la définition d'un faciès régional. Thèse de Doctorat, Université de Rennes I, $434 \mathrm{p}$.

CLIQUET D., 1982 - Les Industries de Saint-Julien de la Liègue. Le Moustérien à petits bifaces dominants. Mémoire de Maîtrise, Université de Paris I / Sorbonne, $177 \mathrm{p}$.
CLIQUET D., 1995 - Les Industries moustériennes à petits bifaces dominants de Haute-Normandie (France). In Les industries à pointes foliacées d'Europe Centrale. Actes du Colloque International de Miskolc (Hongrie), Paléo, supplément n ${ }^{\circ}$ 1, 127-131.

CLIQUET D., HERVIEU G., MARTELIN L., \& MARTELINPODER L., 2003 - Le gisement de Pierrepont (Calvados): un nouveau site à outils bifaciaux. Rapport projet collectif de recherche "Paléolithique de Basse-Normandie», 59-62.

CLIQUET D., \& LAUTRIDOU J.-P., 1988 - Le Moustérien à petits bifaces dominants de Saint-Julien de la Liègue (Eure). In A. Tuffreau (dir.), Cultures et industries en milieu loessique. Actes du Colloque International, Amiens, 1986. Revue Archéologique de Picardie, 1-2, 175-185.

CLIQUET D., LAUTRIDOU J.-P., RIVARD J.-J., ALIX P., GOSSELIN R., \& LORREN P., 2001 - Les industries à outils bifaciaux du Paléolithique moyen en Normandie armoricaine: l'exemple du site de Saint-Brice-sous-Rânes (Orne - France). In D. Cliquet (dir.), Les industries à outils bifaciaux du Paléolithique moyen d'Europe Occidentale. Actes de la table ronde de Caen (France), ERAUL, 98, 93-106.

CLIQUET D., LAUTRIDOU J.-P., LORREN P., MERCIER N., \& RIVARD J.-J., 2005 - Saint-Brice-sous-Rânes «La Bruyère». Rapport de fouille, $24 \mathrm{p}$.

CLIQUET D., MERCIER N., VALLADAS L., FROGET L., MICHEL D., VAN VLIET-LANOË B., \& VILGRAIN G., 2003 Apport de la thermoluminescence sur silex chauffés à la chronologie des sites paléolithiques de Normandie: nouvelles données et interprétation. Quaternaire, 14 (1), 51-64.

COUTARD S., 1998 - Panorama des silex jurassiques bas-normands. Essai de caractérisation et répartition... vers un usage archéologique. Mémoire de Maîtrise, Université de Caen, $75 \mathrm{p}$.

DESCHODT L., FERAY P., \& VALLIN L. avec la collaboration de BOSSUT D., LANTOINE J. et MASSON B., 2006 - Saint-Amandles-Eaux (59) «Bas du Mont des Bruyères». Rapport de diagnostic archéologique, $20 \mathrm{p}$.

DJINDJIAN F., KOSLOWSKI J., \& OTTE M., 1999 - Le Paléolithique supérieur en Europe. Paris, Armand Colin, 474 p.

GAMBLE C., 1999 - The Palaeolithic Societies of Europe. Cambridge University Press, $505 \mathrm{p}$.

HUET B., 2006 - De l'influence des matières premières lithiques sur les comportements techno-économiques au Paléolithique moyen: l'exemple du Massif armoricain. Thèse de Doctorat, Université de Rennes I, $523 \mathrm{p}$.

KUNTZ G., MÉNILLET F., LE GALL J., \& RIOULT M., 1989 Carte géologique et notice explicative de la feuille Argentan $1 / 50000^{e}$. Éd. du BRGM, $99 \mathrm{p}$.

LASSEUR E., 2001 - Approvisionnement en matières premières sur le site paléolithique moyen de Saint-Brice-sous-Rânes. Mémoire de Maîtrise, Université de Caen, $101 \mathrm{p}$.

LASSEUR E., LECLERC F., \& CLIQUET D., 2005 - Accessibilité, acquisition et utilisation de la matière première : étude gîtologique de deux gisements du Paléolithique moyen, la vallée de la Seulles (Calvados) et le site de Saint-Brice-sous-Rânes (Orne). In N. Molines, M.-H. Moncel \& J.-L. Monnier (dir.), Les premiers peuplements en Europe. Actes du colloque international de Rennes, 2003, Hadrian Books (BAR International Series ; 1364), Oxford, 409-417.

LAUTRIDOU J.-P., 1985 - Le Cycle périglaciaire pléistocène en Europe du Nord-Ouest et plus particulièrement en Normandie. Centre de géomorphologie, Caen, $908 \mathrm{p}$.

LHOMME V., 2000 - Industries lithiques du Paléolithique moyen à nombreuses fractures. Les exemples du secteur sud de Champlost (Yonne) et de la couche inférieure de "Chez-Pourré-Chez-Comte» (Corrèze). Thèse, Université des Sciences et Technologies de Lille I, $248 \mathrm{p}$.

LOCHT J.-L. (dir.), 2002 - Bettencourt-Saint-Ouen (Somme). Cinq occupations paléolithiques au début de la dernière glaciation. D.A.F., Editions Maison des Sciences de l'Homme, Paris, $\mathrm{n}^{\circ} \mathbf{9 0}$, $176 \mathrm{p}$.

LOCHT J.-L., ANTOINE P., BAHAIN J.-J., DRWILA G., RAYMOND P., LIMONDIN-LOZOUET N., GAUTHIER A., DEBENHAM N., FRECHEN M., ROUSSEAU D.-D., HATTE C., HAESAERTS P., \& METSDAGH H., 2003 - Le gisement paléolithique moyen et les séquences pléistocènes de Villiers-Adam (Val d'Oise). Chronostratigraphie, environnements et occupations humaines. Gallia préhistoire, 45, 1-111.

MERCIER N., VALLADAS H., \& VALLADAS G., 1992 - Some observations on palaeodose determination in burnt flints. Ancient $T L, \mathrm{n}^{\circ} \mathbf{3}, 28-32$.

MERCIER N., FROGET L., MiALlier D., PILleYRE T., SANZELLE S., \& TRIBOLO C., 2004 - Nouvelles données chro- 
nologiques pour le site de Menez-Dregan 1 (Bretagne): l'apport de la thermoluminescence. Quaternaire, 15, 253-261.

MONNIER J.-L., 1980 - Le Paléolithique de la Bretagne dans son cadre géologique. Rennes, Travaux du Laboratoire d'Anthropologie, Préhistoire, Protohistoire et Quaternaire Armoricain, C.N.R.S., $607 \mathrm{p}$.

MONNIER J.-L., 1989 - Le gisement paléolithique moyen et supérieur de Karreg-Ar-Yellan (Ploubazlanec, Côtes-du-Nord). Gallia, 31, 1-21.

OTTE M., 1996 - Le paléolithique inférieur et moyen en Europe. Paris, Armand Colin, $297 \mathrm{p}$.

PINOIT L., 2001 - Analyse typo-technologique du gisement de BoisL'Abbé (Saint-Julien-de-la-Liègue, Eure). In D. Cliquet (dir.), Les industries à outils bifaciaux du Paléolithique moyen d'Europe Occidentale. Actes de la table ronde de Caen, ERAUL, 98, 85-91.

RICHTER J., 2006 - Neanderthals in their lanscape. In B. Demrsin \& M. Otte (dir.), Neanderthals in Europe. Proceedings of the International Conference held in the Gallo-Roman Museum in Tongeren, September 17-19th 2004, ERAUL, 117, 56-61.

SORESSI M., 2002 - Le Moustérien de tradition acheuléenne du sudouest de la France. Discussion sur la signification du faciès à partir de l'étude comparée de quatre sites: Pech-de-l'Azé I, Le Moustier La Rochette et la Grotte XVI. Thèse de doctorat, Université Bordeaux I, $330 \mathrm{p}$.

VALLADAS H., 1992 - Thermoluminescence dating of flint. Quaternary Science Reviews, 11, 1-5. 